\title{
EXACT CONTROLLABILITY IN FLUID - SOLID STRUCTURE: THE HELMHOLTZ MODEL *
}

\author{
JeAn-PierRe RAymond ${ }^{1}$ And Muthusamy VAnNinathan ${ }^{2}$
}

\begin{abstract}
A model representing the vibrations of a fluid-solid coupled structure is considered. Following Hilbert Uniqueness Method (HUM) introduced by Lions, we establish exact controllability results for this model with an internal control in the fluid part and there is no control in the solid part. Novel features which arise because of the coupling are pointed out. It is a source of difficulty in the proof of observability inequalities, definition of weak solutions and the proof of controllability results.
\end{abstract}

Mathematics Subject Classification. 35B37, 93C20.

Received August 20, 2003. Revised March 1, 2004.

\section{INTRODUCTION}

In this paper we are interested in the controllability of a fluid-solid structure model introduced in [4] and [5] which is written down below. Assuming that the fluid is inviscid and its movements are two-dimensional, infinitesimal and given by a velocity potential, the model couples them with the motion of a solid represented by a harmonic oscillator. The coupling is in two places: the fluid exerts a pressure force on the solid through the interface and the solid, in turn, influences the fluid motion via the interface condition which requires that the normal component of the fluid and solid velocities coincide.

Let $\mathcal{O}$ be a simply connected bounded domain in $\mathbb{R}^{2}$ with a regular boundary $\Gamma_{e}$, and let $S$ an open and regular subset in $\mathcal{O}$, of boundary $\Gamma$. We suppose that $\bar{S} \subset \mathcal{O}$, and we set $\Omega=\mathcal{O} \backslash \bar{S}$. We consider a fluid occupying the open set $\Omega$ and a solid occupying the closed set $\bar{S}$ described by the following coupled system

$$
\begin{aligned}
& \phi^{\prime \prime}-\Delta \phi=u \quad \text { in } Q, \\
& \phi=0 \quad \text { on } \Sigma_{e} \text {, } \\
& \partial_{n} \phi=s^{\prime} \cdot n \quad \text { on } \Sigma \text {, } \\
& \phi(0)=\phi^{0} \quad \text { and } \quad \phi^{\prime}(0)=\phi^{1} \quad \text { in } \Omega \text {, } \\
& s^{\prime \prime}+s=-\int_{\Gamma} \phi^{\prime} n \quad \text { in }(0, T), \\
& s(0)=s^{0} \quad \text { and } \quad s^{\prime}(0)=s^{1} \quad \text { in } \quad \mathbb{R}^{2} .
\end{aligned}
$$

Keywords and phrases. Fluid - solid structure, exact controllability.

* The second author gratefully acknowledges the support received from DRDO-IISc Joint Programme on Advanced Research in Mathematical Engineering. This work forms part of the above Programme.

${ }^{1}$ Université Paul Sabatier, UMR 5640, Laboratoire MIP, 31062 Toulouse Cedex 4, France;

raymond@mip.ups-tlse.fr

2 IISc-TIFR Mathematics Programme, TIFR Centre, Bangalore 560012, India. 
In this setting $Q=\Omega \times(0, T), T>0, \Sigma_{e}=\Gamma_{e} \times(0, T), \Sigma=\Gamma \times(0, T)$. The forcing term $u$ in the fluid region is a function used to control the fluid-solid structure system. The function $\phi$ is the velocity potential in the fluid, $s \in \mathbb{R}^{2}$ denotes the displacement of the solid and $n$ is the unit normal on $\Gamma$ exterior to $\Omega$.

The main objective of this paper is to extend the well known internal controllability results of the wave equation [10], Theorems 2.1 and 2.3, Chapter 7 to the system (1). For simplicity we have taken a control $u$ acting everywhere in the domain $\Omega$, and in this case we can obtain controllability results for any time $T>0$. However we think that the results obtained in this paper may be extended to a localized internal control and to a boundary control, with appropriate geometrical conditions. This will be done in a forthcoming paper. In the present paper by considering the simplest case corresponding to a distributed control acting in $\Omega$, we want to focus our analysis on the difficulties coming from the coupling between the wave equation and the oscillator equation. Indeed the term $\int_{\Gamma} \phi^{\prime} n$ in the oscillator equation is a source of difficulties in the treatment of the observability inequalities, in the definition of weak solutions, and in the proofs of controllability results.

Let us explain in few words what are the main ingredients to overcome these problems. The Hilbert Uniqueness Method, due to Lions [10], consists in constructing an isomorphism $\Lambda$ from a Hilbert space $F$ onto $F^{\prime}$, where $F$ is the closure of a space of regular functions for the norm $\|\cdot\|_{F}$ appearing in an observability inequality, and $F^{\prime}$ is the space of initial data which are controllable. In the standard HUM, we have

$$
\langle\Lambda \Phi, \Phi\rangle_{F^{\prime}, F}=\|\Phi\|_{F}^{2},
$$

for all $\Phi \in F$, and $\Lambda=\Lambda^{*}$.

Let us first consider the case of controllability of problem (1) with initial data $\left(\phi^{1}, \phi^{0}, s^{1}, s^{0}\right)$ belonging to $F^{\prime}=L^{2}(\Omega) \times V \times \mathbb{R}^{2} \times \mathbb{R}^{2}$, where $V=\left\{\phi \in H^{1}(\Omega) \mid \phi=0\right.$ on $\left.\Gamma_{e}\right\}$. But contrarily to the wave equation, the operator $\Lambda$ corresponding to system (1) cannot be defined on $F=L^{2}(\Omega) \times V^{\prime} \times \mathbb{R}^{2} \times \mathbb{R}^{2}$, because weak solutions of (1) are not well defined for initial data $\left(\phi^{0}, \phi^{1}, s^{0}, s^{1}\right)$ in $F$. First we have to identify the correct space $Y$ for which weak solutions are well defined (see Sect. 3). Next we define $\Lambda$ on $Z=V \times L^{2}(\Omega) \times \mathbb{R}^{2} \times \mathbb{R}^{2}$, and we prove that its extension $\widetilde{\Lambda}$ to $Y$ is an isomorphism from $Y$ onto $F^{\prime}$. But contrarily to the wave equation the operator $\widetilde{\Lambda}$ is not selfadjoint and (2) is replaced by

$$
\langle\widetilde{\Lambda}(\Phi, \mathbf{s}), \widetilde{J}(\Phi, \mathbf{s})\rangle_{F^{\prime}, F}=\|(\Phi, \mathbf{s})\|_{Y}^{2},
$$

for all initial data $\left(\phi^{0}, \phi^{1}, s^{0}, s^{1}\right)=(\Phi, \mathbf{s}) \in Y$, or equivalently by

$$
\left\langle\widetilde{J}^{*} \widetilde{\Lambda}(\Phi, \mathbf{s}),(\Phi, \mathbf{s})\right\rangle_{F^{\prime}, F}=\|(\Phi, \mathbf{s})\|_{Y}^{2},
$$

where $\widetilde{J}$ is an isomorphism from $Y$ to $F$, which for regular data $\left(\phi^{0}, \phi^{1}, s^{0}, s^{1}\right) \in Z$ is given by

$$
\widetilde{J}\left(\phi^{0}, \phi^{1}, s^{0}, s^{1}\right)=\left(\phi^{0}, \phi^{1}, s^{0}, s^{1}+\int_{\Gamma} \phi^{0} n\right) .
$$

Thanks to these ingredients, we can adapt the Hilbert Uniqueness Method to sytem (1). To control weak solutions, that is solutions with initial data in $Y$, the construction is a little bit different from the previous one (see Sects. 6 and 7).

We think that the approach introduced here may be helpful to tackle the control of more complex fluid-solid structure models such as those considered in $[1,8,9,11]$. A first progress in that direction is carried out in [6].

\section{Notation AND Statement of the main Result}

Let $V$ be the space defined by

$$
V=\left\{\phi \in H^{1}(\Omega) \mid \phi=0 \quad \text { on } \quad \Gamma_{e}\right\},
$$


and denote by $V^{\prime}$ the topological dual of $V$. The space $V$ will be equipped with the norm

$$
\phi \longmapsto\left(\int_{\Omega}|\nabla \phi|^{2} \mathrm{~d} x\right)^{\frac{1}{2}}
$$

The norm in $V$ will be denoted by $\|\cdot\|_{V}$. The same kind of notation will be used for other Banach spaces. The norm in $\mathbb{R}^{2}$ will be simply denoted by $|\cdot|$. The inner product of $s \in \mathbb{R}^{2}$ and $\sigma \in \mathbb{R}^{2}$ is denoted by $s \cdot \sigma$.

The first main result of the paper is stated in the following theorem. It is a controllability result with internal controls. Throughout the paper we fix $T>0$ arbitrary.

Theorem 1.1. For all $\phi^{0} \in V, \phi^{1} \in L^{2}(\Omega), s^{0} \in \mathbb{R}^{2}, s^{1} \in \mathbb{R}^{2}$, there exists a function $u \in C\left([0, T] ; L^{2}(\Omega)\right)$ such that the solution to equation (1) obeys

$$
\phi(T)=\phi^{\prime}(T)=0 \quad \text { and } \quad s(T)=s^{\prime}(T)=0 .
$$

In the context of Theorem 1.1, we have finite energy solutions for equation (1), whereas in Section 3 a concept of weak solutions is introduced and studied. The second main result of the paper is stated in Theorem 6.1. It is a controllability result for weak solutions introduced in Section 3.

\section{Definition and Regularity of solutions to the CONTRolled System}

In this section, we present estimates for finite energy solutions of equation (1) where we have introduced a forcing term $v$ on the solid part:

$$
\begin{array}{ll}
\phi^{\prime \prime}-\Delta \phi=u & \text { in } Q, \\
\phi=0 & \text { on } \Sigma_{e}, \\
\partial_{n} \phi=s^{\prime} \cdot n & \text { on } \Sigma, \\
\phi(0)=\phi^{0} \quad \text { and } \quad \phi^{\prime}(0)=\phi^{1} & \text { in } \Omega, \\
s^{\prime \prime}+s=-\int_{\Gamma} \phi^{\prime} n+v & \text { in }(0, T), \\
s(0)=s^{0} \quad \text { and } \quad s^{\prime}(0)=s^{1} & \text { in } \mathbb{R}^{2} .
\end{array}
$$

Theorem 2.1. (i) For all $\left(\phi^{0}, \phi^{1}, s^{0}, s^{1}\right) \in V \times L^{2}(\Omega) \times \mathbb{R}^{2} \times \mathbb{R}^{2}$, and $(u, v) \in L^{1}\left(0, T ; L^{2}(\Omega)\right) \times L^{1}\left(0, T ; \mathbb{R}^{2}\right)$, the equation (3) admits a unique solution $(\phi, s)$ in $\left(C([0, T] ; V) \cap C^{1}\left([0, T] ; L^{2}(\Omega)\right)\right) \times C^{1}\left([0, T] ; \mathbb{R}^{2}\right)$, which is called a finite energy solution, and

$$
\begin{aligned}
\|\phi\|_{C([0, T] ; V)}+\left\|\phi^{\prime}\right\|_{C\left([0, T] ; L^{2}(\Omega)\right)}+\| & s \|_{C^{1}\left([0, T] ; \mathbb{R}^{2}\right)} \\
& \leq C\left(\left\|\phi^{0}\right\|_{V}+\left\|\phi^{1}\right\|_{L^{2}(\Omega)}+\left|s^{0}\right|+\left|s^{1}\right|+\|u\|_{L^{1}\left(0, T ; L^{2}(\Omega)\right)}+\|v\|_{L^{1}\left(0, T ; \mathbb{R}^{2}\right)}\right) .
\end{aligned}
$$

(ii) If $u=v=0$ in (3), then the solution possesses an additional property namely $\phi^{\prime \prime} \in C\left([0, T] ; V^{\prime}\right)$ and

$$
\left\|\phi^{\prime \prime}\right\|_{C\left([0, T] ; V^{\prime}\right)} \leq C\left(\left\|\phi^{0}\right\|_{V}+\left\|\phi^{1}\right\|_{L^{2}(\Omega)}+\left|s^{0}\right|+\left|s^{1}\right|\right) .
$$

Proof. (i) Setting $z=\left(\phi, \phi^{\prime}, s, s^{\prime}\right)$, equation (3) may be written in the form of a first order system

$$
\frac{\mathrm{d} z}{\mathrm{~d} t}=A z+U, \quad z(0)=z_{0}
$$


with

$$
A z=A\left(\begin{array}{c}
\phi_{0} \\
\phi_{1} \\
s_{0} \\
s_{1}
\end{array}\right)=\left(\begin{array}{c}
\phi_{1} \\
\Delta \phi_{0} \\
s_{1} \\
-s_{0}-\int_{\Gamma} \phi_{1} n
\end{array}\right), \quad U=\left(\begin{array}{c}
0 \\
u \\
0 \\
v
\end{array}\right), \quad \text { and } \quad z_{0}=\left(\begin{array}{c}
\phi^{0} \\
\phi^{1} \\
s^{0} \\
s^{1}
\end{array}\right)
$$

Set $Z=V \times L^{2}(\Omega) \times \mathbb{R}^{2} \times \mathbb{R}^{2}$. The domain of $A$ in $Z$ is

$$
D(A)=\left\{\left(\phi_{0}, \phi_{1}, s_{0}, s_{1}\right) \in Z \mid \phi_{0} \in H^{2}(\Omega), \partial_{n} \phi_{0}=s_{1} \cdot n \text { on } \Gamma, \phi_{1} \in V\right\}
$$

The Hilbert space $Z$ is equipped with the inner product defined by

$$
\left(\left(\phi_{0}, \phi_{1}, s_{0}, s_{1}\right),\left(\psi_{0}, \psi_{1}, \sigma_{0}, \sigma_{1}\right)\right)_{Z}=\int_{\Omega} \nabla \phi_{0} \nabla \psi_{0}+\int_{\Omega} \phi_{1} \psi_{1}+s_{0} \cdot \sigma_{0}+s_{1} \cdot \sigma_{1}
$$

It is clear that the operator $(A, D(A))$ is a closed operator in $Z$, and that the domain $D(A)$ is dense in $Z$.

For every $\left(\phi_{0}, \phi_{1}, s_{0}, s_{1}\right) \in D(A)$ we have

$$
\left(A\left(\phi_{0}, \phi_{1}, s_{0}, s_{1}\right),\left(\phi_{0}, \phi_{1}, s_{0}, s_{1}\right)\right)_{Z}=\int_{\Omega} \nabla \phi_{1} \nabla \phi_{0}+\int_{\Omega} \Delta \phi_{0} \phi_{1}+s_{1} \cdot s_{0}-\left(s_{0}+\int_{\Gamma} \phi_{1} n\right) \cdot s_{1}=0 .
$$

Thus, in particular, $(A, D(A))$ is a dissipative operator in $Z$.

We can easily verify that the adjoint operator of $A$ is defined by $D\left(A^{*}\right)=D(A)$ and $A^{*}=-A$. Therefore $(A, D(A))$ and $\left(A^{*}, D\left(A^{*}\right)\right)$ are both m-dissipative operators on $Z$ and $(A, D(A))$ is skew-adjoint. From Stone's theorem, it follows that the operator $(A, D(A))$ is the infinitesimal generator of a strongly continuous unitary group on $Z$. The end of proof follows from [3] (Chap. 1, Prop. 3.2).

(ii) To prove the part (ii), we remark that the system can also be written equivalently in the following integral form:

For all $v \in V$, the mapping

$$
t \longmapsto \int_{\Omega} \phi(t) v
$$

belongs to $H^{2}(0, T)$ and satisfies

$$
\begin{gathered}
\frac{\mathrm{d}^{2}}{\mathrm{~d} t^{2}} \int_{\Omega} \phi(t) v+\int_{\Omega} \nabla \phi(t) \cdot \nabla v=\frac{\mathrm{d} s}{\mathrm{~d} t} \cdot \int_{\Gamma} v n \quad \text { in }(0, T), \\
\left.\int_{\Omega} \phi(t) v\right|_{t=0}=\int_{\Omega} \phi^{0} v,\left.\quad \frac{\mathrm{d}}{\mathrm{d} t} \int_{\Omega} \phi(t) v\right|_{t=0}=\int_{\Omega} \phi^{1} v \\
\frac{\mathrm{d}^{2}}{\mathrm{~d} t^{2}} s(t)+s(t)=-\frac{\mathrm{d}}{\mathrm{d} t} \int_{\Gamma} \phi(t) n \quad \text { in }(0, T) \\
s(0)=s^{0}, \quad \frac{\mathrm{d} s}{\mathrm{~d} t}(0)=s^{1} .
\end{gathered}
$$

From the first relation, using that $\phi \in C([0, T] ; V)$ and $s \in C^{1}\left([0, T] ; \mathbb{R}^{2}\right)$, we deduce the required result.

Remark 2.2. Observe that, when the assumptions of Theorem 2.1 are satisfied, the solution $(\phi, s)$ to equation (3) satisfies the following energy identity

$$
E(t)=E(0)+\int_{0}^{t} \int_{\Omega} u \phi^{\prime} \mathrm{d} x \mathrm{~d} \tau+\int_{0}^{t} v \cdot s^{\prime} \mathrm{d} \tau \quad \text { for all } t \in[0, T]
$$


where

$$
2 E(t)=\int_{\Omega}\left(\phi^{\prime}(t)\right)^{2} \mathrm{~d} x+\int_{\Omega}|\nabla \phi(t)|^{2} \mathrm{~d} x+\left|s^{\prime}(t)\right|^{2}+|s(t)|^{2} .
$$

In particular, if $u=0$ and $v=0$ then the energy $E(t)$ is constant.

Remark 2.3. In the context of part (ii) of the above theorem, we cannot assert $s^{\prime \prime} \in C\left([0, T] ; \mathbb{R}^{2}\right)$. This is one of the effects of the coupling.

Theorem 2.4. For all $\left(\phi^{0}, \phi^{1}, s^{0}, s^{1}\right) \in D(A)$, and $u=0$, equation (1) admits a unique strong solution $(\phi, s)$ in $\left(C\left([0, T] ; H^{2}(\Omega)\right) \cap C^{1}([0, T] ; V)\right) \times C^{2}\left([0, T] ; \mathbb{R}^{2}\right)$ and

$$
\|\phi\|_{C\left([0, T] ; H^{2}(\Omega)\right)}+\|\phi\|_{C^{1}([0, T] ; V)}+\|s\|_{C^{2}\left([0, T] ; \mathbb{R}^{2}\right)} \leq C\left(\left\|\phi^{0}\right\|_{H^{2}(\Omega)}+\left\|\phi^{1}\right\|_{V}+\left|s^{0}\right|+\left|s^{1}\right|\right) .
$$

Proof. Since $\left(\phi^{0}, \phi^{1}, s^{0}, s^{1}\right) \in D(A)$, it follows that the solution $z=\left(\phi, \phi^{\prime}, s, s^{\prime}\right)$ to equation (1) belongs to $C([0, T] ; D(A))[3]$ (Chap. 1, Prop. 3.3), and

$$
\|z\|_{C([0, T] ; D(A))} \leq C\left\|\left(\phi^{0}, \phi^{1}, s^{0}, s^{1}\right)\right\|_{D(A)} .
$$

The proof is complete.

Now we state a theorem that will be useful to prove estimates by the transposition method, estimates which are necessary for the control of weak solutions (see Th. 6.9).

Theorem 2.5. Consider the finite energy solution $(\phi, s)$ to the system (3) with $\left(\phi^{0}, \phi^{1}, s^{0}, s^{1}\right)=0$.

(i) For all $u=f_{1}^{\prime}$ with $f_{1} \in C_{c}^{1}((0, T] ; V)$ and for $v=0$, we have

$$
\left\|\phi^{\prime}\right\|_{L^{1}\left(0, T ; L^{2}(\Omega)\right)}+\|\phi\|_{L^{\infty}(0, T ; V)}+\left\|s^{\prime}\right\|_{L^{\infty}\left(0, T ; \mathbb{R}^{2}\right)}+\|s\|_{L^{\infty}\left(0, T ; \mathbb{R}^{2}\right)} \leq C\left\|f_{1}\right\|_{L^{1}(0, T ; V)} .
$$

(ii) For $u=0$ and $v=v_{1}^{\prime}$ with $v_{1} \in C_{c}^{1}\left((0, T] ; \mathbb{R}^{2}\right)$, we have

$$
\left\|\phi^{\prime}\right\|_{L^{\infty}\left(0, T ; L^{2}(\Omega)\right)}+\|\phi\|_{L^{\infty}(0, T ; V)}+\left\|s^{\prime}\right\|_{L^{1}\left(0, T ; \mathbb{R}^{2}\right)}+\|s\|_{L^{\infty}\left(0, T ; \mathbb{R}^{2}\right)} \leq C\left\|v_{1}\right\|_{L^{1}\left(0, T ; \mathbb{R}^{2}\right)} .
$$

Proof. (i) We set $w(t)=\int_{0}^{t} \phi$ and $\xi(t)=\int_{0}^{t} s$. The pair $(w, \xi)$ is the solution to the system

$$
\begin{aligned}
& w^{\prime \prime}-\Delta w=f_{1} \quad \text { in } Q, \\
& w=0 \quad \text { on } \Sigma_{e} \text {, } \\
& \partial_{n} w=\xi^{\prime} \cdot n \quad \text { on } \Sigma, \\
& w(0)=0 \quad \text { and } \quad w^{\prime}(0)=0 \quad \text { in } \Omega \text {, } \\
& \xi^{\prime \prime}+\xi=-\int_{\Gamma} w^{\prime} n \quad \text { in }(0, T) \text {, } \\
& \xi(0)=0 \quad \text { and } \quad \xi^{\prime}(0)=0 \quad \text { in } \mathbb{R}^{2} \text {. }
\end{aligned}
$$

We multiply by $\left(-\Delta w^{\prime}\right)$ the equation satisfied by $w$, with integrations by parts and a Green formula we obtain

$$
\begin{aligned}
\int_{\Omega} f_{1}\left(-\Delta w^{\prime}\right) & =\int_{\Omega} \nabla f_{1} \nabla w^{\prime}-\int_{\Gamma} \partial_{n} w^{\prime} f_{1}=\int_{\Omega} \nabla f_{1} \nabla w^{\prime}-\int_{\Gamma} \xi^{\prime \prime} \cdot n f_{1} \\
& =\int_{\Omega} \nabla f_{1} \nabla w^{\prime}+\int_{\Gamma} \xi \cdot n f_{1}+\int_{\Gamma} w^{\prime} n \cdot \int_{\Gamma} f_{1} n
\end{aligned}
$$

and

$$
\int_{\Omega} w^{\prime \prime}\left(-\Delta w^{\prime}\right)=\int_{\Omega} \nabla w^{\prime \prime} \cdot \nabla w^{\prime}-\int_{\Gamma} \partial_{n} w^{\prime} w^{\prime \prime} .
$$


Since

we have

$$
-\int_{\Gamma} \partial_{n} w^{\prime} w^{\prime \prime}=-\int_{\Gamma} \xi^{\prime \prime} \cdot n w^{\prime \prime}=\int_{\Gamma} w^{\prime} n \cdot \int_{\Gamma} w^{\prime \prime} n+\int_{\Gamma} \xi \cdot n w^{\prime \prime}
$$

$$
\begin{aligned}
\int_{\Omega}\left(w^{\prime \prime}-\Delta w\right)\left(-\Delta w^{\prime}\right) & =\int_{\Omega} \nabla w^{\prime \prime} \cdot \nabla w^{\prime}+\int_{\Omega} \Delta w \Delta w^{\prime}+\int_{\Gamma} w^{\prime} n \cdot \int_{\Gamma} w^{\prime \prime} n+\int_{\Gamma} \xi \cdot n w^{\prime \prime} \\
& =\int_{\Omega} \nabla f_{1} \nabla w^{\prime}+\int_{\Gamma} \xi \cdot n f_{1}+\int_{\Gamma} w^{\prime} n \cdot \int_{\Gamma} n f_{1} .
\end{aligned}
$$

Integrating between 0 and $t$ we obtain

$$
\begin{array}{r}
\frac{1}{2}\left(\left\|\nabla w^{\prime}(t)\right\|_{L^{2}(\Omega)}^{2}+\|\Delta w(t)\|_{L^{2}(\Omega)}^{2}+\left|\int_{\Gamma} w^{\prime}(t) n\right|^{2}\right)=\int_{0}^{t} \int_{\Omega} \nabla f_{1} \cdot \nabla w^{\prime} \\
+\int_{0}^{t} \int_{\Gamma} \xi \cdot n f_{1}+\int_{0}^{t}\left(\int_{\Gamma} w^{\prime} n \cdot \int_{\Gamma} f_{1} n\right) \\
+\int_{0}^{t} \int_{\Gamma} \xi^{\prime} \cdot n w^{\prime}-\int_{\Gamma} \xi(t) \cdot n w^{\prime}(t) . \quad(7)
\end{array}
$$

Now multiplying by $\xi^{\prime}$ the equation satisfied by $\xi$. Integrating between 0 and $t$ we obtain

$$
\left|\xi^{\prime}(t)\right|^{2}+|\xi(t)|^{2}=-2 \int_{0}^{t} \int_{\Gamma} w^{\prime} n \cdot \xi^{\prime}
$$

Combining (7) and (8) we arrive at the equality

$$
\begin{aligned}
\left\|\nabla w^{\prime}(t)\right\|_{L^{2}(\Omega)}^{2}+\|\Delta w(t)\|_{L^{2}(\Omega)}^{2}+\left|\xi^{\prime}(t)\right|^{2}+\left|\xi(t)+\int_{\Gamma} w^{\prime}(t) n\right|^{2} & =2 \int_{0}^{t} \int_{\Omega} \nabla f_{1} \cdot \nabla w^{\prime} \\
& +2 \int_{0}^{t} \int_{\Gamma} \xi \cdot n f_{1}+2 \int_{0}^{t}\left(\int_{\Gamma} w^{\prime} n \cdot \int_{\Gamma} f_{1} n\right) .
\end{aligned}
$$

Using a trace theorem and Hölder's inequality we first deduce the estimate

$$
\begin{aligned}
\left\|\nabla w^{\prime}(t)\right\|_{L^{2}(\Omega)}^{2}+\|\Delta w(t)\|_{L^{2}(\Omega)}^{2}+\left|\xi(t)+\int_{\Gamma} w^{\prime}(t) n\right|^{2}+ & \left|\xi^{\prime}(t)\right|^{2} \\
& \leq C\left\|f_{1}\right\|_{L^{1}(0, T ; V)}\left(\left\|\nabla w^{\prime}\right\|_{L^{\infty}\left(0, T ; L^{2}(\Omega)\right)}+\|\xi\|_{L^{\infty}\left(0, T ; \mathbb{R}^{2}\right)}\right) .
\end{aligned}
$$

Since $\xi(0)=0$ and $\left|\xi^{\prime}(t)\right|^{2}$ is bounded by $C\left\|f_{1}\right\|_{L^{1}(0, T ; V)}\left(\left\|\nabla w^{\prime}\right\|_{L^{\infty}\left(0, T ; L^{2}(\Omega)\right)}+\|\xi\|_{L^{\infty}\left(0, T ; \mathbb{R}^{2}\right)}\right)$, we can obtain the estimate

$$
|\xi(t)|^{2} \leq C\left\|f_{1}\right\|_{L^{1}(0, T ; V)}\left(\left\|\nabla w^{\prime}\right\|_{L^{\infty}\left(0, T ; L^{2}(\Omega)\right)}+\|\xi\|_{L^{\infty}\left(0, T ; \mathbb{R}^{2}\right)}\right) .
$$

With the above estimates and Young's inequality we finally prove

$$
\left\|\nabla w^{\prime}(t)\right\|_{L^{2}(\Omega)}^{2}+\|\Delta w(t)\|_{L^{2}(\Omega)}^{2}+\left|\int_{\Gamma} w^{\prime}(t) n\right|^{2}+\left|\xi^{\prime}(t)\right|^{2}+|\xi(t)|^{2} \leq C\left\|f_{1}\right\|_{L^{1}(0, T ; V)}^{2} .
$$

Since $\left\|\nabla w^{\prime}(t)\right\|_{L^{2}(\Omega)}=\|\phi(t)\|_{V},\left|\xi^{\prime}(t)\right|=|s(t)|$ and $|\xi(t)|=\left|\int_{\Gamma} w^{\prime}(t) n+s^{\prime}(t)\right|=\left|\int_{\Gamma} \phi(t) n+s^{\prime}(t)\right|$, we obtain

$$
\|\phi\|_{L^{\infty}(0, T ; V)}+\left\|s^{\prime}\right\|_{L^{\infty}\left(0, T ; \mathbb{R}^{2}\right)}+\|s\|_{L^{\infty}\left(0, T ; \mathbb{R}^{2}\right)} \leq C\left\|f_{1}\right\|_{L^{1}(0, T ; V)} .
$$

Next, from the estimate

$$
\|\Delta w(t)\|_{L^{2}(\Omega)} \leq C\left\|f_{1}\right\|_{L^{1}(0, T ; V)}
$$


and the inequality

$$
\left\|\phi^{\prime}(t)\right\|_{L^{2}(\Omega)} \leq\left\|f_{1}(t)\right\|_{L^{2}(\Omega)}+\|\Delta w(t)\|_{L^{2}(\Omega)}
$$

it yields

$$
\left\|\phi^{\prime}\right\|_{L^{1}\left(0, T ; L^{2}(\Omega)\right)} \leq C\left\|f_{1}\right\|_{L^{1}(0, T ; V)} .
$$

(ii) Introducing $w$ and $\xi$ as in part (i), we can find the system satisfied by them. Once again, by using the same multipliers as before, we deduce that

$$
\left\|\nabla w^{\prime}(t)\right\|_{L^{2}(\Omega)}^{2}+\|\Delta w(t)\|_{L^{2}(\Omega)}^{2}+\left|\xi^{\prime}(t)\right|^{2}+\left|\xi(t)+\int_{\Gamma} w^{\prime}(t) n\right|^{2}=2 \int_{0}^{t} \xi^{\prime}(\tau) v_{1}(\tau) \mathrm{d} \tau .
$$

The estimate announced in part (ii) of the theorem follows from this. The proof is complete.

Remark 2.6. Estimate (5) is proved for all $f_{1} \in C_{c}^{1}((0, T] ; V)$, even if it is only needed for all $f_{1} \in C_{c}^{1}((0, T) ; V)$ (see the proof of Th. 6.3). By using functions $f_{1}$ belonging to $C_{c}^{1}((0, T] ; V)$, we may observe that we cannot obtain the estimate

$$
\left\|\phi^{\prime}\right\|_{L^{\infty}\left(0, T ; L^{2}(\Omega)\right)} \leq C\left\|f_{1}\right\|_{L^{1}(0, T ; V)} .
$$

Indeed, if it was true, we could write

$$
\left\|f_{1}(T)\right\|_{L^{2}(\Omega)}=\left\|w^{\prime \prime}(T)-\Delta w(T)\right\|_{L^{2}(\Omega)} \leq\left\|\phi^{\prime}(T)\right\|_{L^{2}(\Omega)}+\|\Delta w(T)\|_{L^{2}(\Omega)} \leq C\left\|f_{1}\right\|_{L^{1}(0, T ; V)},
$$

for all $f_{1} \in C_{c}^{1}((0, T] ; V)$, which is obviously wrong.

For similar reasons we cannot prove the estimate

$$
\left\|\phi^{\prime}\right\|_{L^{2}\left(0, T ; L^{2}(\Omega)\right)} \leq C\left\|f_{1}\right\|_{L^{1}(0, T ; V)} .
$$

Indeed, if it was true, we could write

$$
\left\|f_{1}(t)\right\|_{L^{2}(\Omega)} \leq\left\|\phi^{\prime}(t)\right\|_{L^{2}(\Omega)}+\|\Delta w(t)\|_{L^{2}(\Omega)},
$$

and consequently

$$
\left\|f_{1}\right\|_{L^{2}\left(0, T ; L^{2}(\Omega)\right)} \leq\left\|\phi^{\prime}\right\|_{L^{2}\left(0, T ; L^{2}(\Omega)\right)}+\|\Delta w\|_{L^{2}\left(0, T ; L^{2}(\Omega)\right)} \leq C\left\|f_{1}\right\|_{L^{1}(0, T ; V)},
$$

for all $f_{1} \in C_{c}^{1}((0, T] ; V)$, which is obviously wrong. See the remark after the proof of Theorem 6.9.

Before ending this section, we state a Green formula for finite energy solutions to equation to (3) and finite energy solutions of the following equation

$$
\begin{array}{ll}
\psi^{\prime \prime}-\Delta \psi=f & \text { in } Q, \\
\psi=0 & \text { on } \Sigma_{e}, \\
\partial_{n} \psi=\sigma^{\prime} \cdot n & \text { on } \Sigma, \\
\psi(T)=0 \quad \text { and } \quad \psi^{\prime}(T)=0 & \text { in } \Omega, \\
\sigma^{\prime \prime}+\sigma=-\int_{\Gamma} \psi^{\prime} n+g & \text { in }(0, T), \\
\sigma(T)=0 \quad \text { and } \quad \sigma^{\prime}(T)=0 & \text { in } \mathbb{R}^{2} .
\end{array}
$$


Theorem 2.7. The finite energy solution $(\phi, s)$ of equation (3) and the finite energy solution $(\psi, \sigma)$ of equation (10) satisfy the formula

$$
\begin{aligned}
\int_{Q} f \phi+\int_{0}^{T} g \cdot s=\int_{Q} \psi u+\int_{0}^{T} v \cdot \sigma & -\left\langle\phi^{0}, \psi^{\prime}(0)\right\rangle_{V, V^{\prime}} \\
& +\left(\psi(0), \phi^{1}\right)_{L^{2}(\Omega)}+\sigma(0) \cdot\left(s^{1}+\int_{\Gamma} \phi^{0} n\right)-s^{0} \cdot\left(\sigma^{\prime}(0)+\int_{\Gamma} \psi(0) n\right)
\end{aligned}
$$

for all $\left(\phi^{0}, \phi^{1}, s^{0}, s^{1}\right) \in Z,(u, v) \in L^{1}\left(0, T ; L^{2}(\Omega)\right) \times L^{1}\left(0, T ; \mathbb{R}^{2}\right),(f, g) \in L^{1}\left(0, T ; L^{2}(\Omega)\right) \times L^{1}\left(0, T ; \mathbb{R}^{2}\right)$.

Proof. The proof is classical and is left to the reader.

\section{WEAK SOLUTIONS}

As explained in the introduction, weak solutions of system (1) cannot be well defined for arbitrary initial data $\left(\phi^{0}, \phi^{1}, s^{0}, s^{1}\right) \in L^{2}(\Omega) \times V^{\prime} \times \mathbb{R}^{2} \times \mathbb{R}^{2}$. A classical method to define weak solutions for rough data consists in using the so-called transposition method. Consider $\left(\phi^{0}, \phi^{1}, s^{0}, s^{1}\right)=\left(0, \phi^{1}, s^{0}, s^{1}\right)$ with $\left(\phi^{1}, s^{0}, s^{1}\right) \in$ $V^{\prime} \times \mathbb{R}^{2} \times \mathbb{R}^{2}$ and $u=0$. In this case, we can say that $(\phi, s) \in L^{\infty}\left(0, T ; L^{2}(\Omega)\right) \times L^{\infty}\left(0, T ; \mathbb{R}^{2}\right)$ is a weak solution of equation (1) if formula (11) with $\left(\phi^{0}, \phi^{1}, s^{0}, s^{1}\right)=\left(0, \phi^{1}, s^{0}, s^{1}\right)$ and $u=0$ is satisfied for every $(f, g) \in L^{1}\left(0, T ; L^{2}(\Omega)\right) \times L^{1}\left(0, T ; \mathbb{R}^{2}\right)$, that is if:

$$
\int_{Q} f \phi+\int_{0}^{T} g \cdot s=\left\langle\psi(0), \phi^{1}\right\rangle_{V, V^{\prime}}+\sigma(0) \cdot s^{1}-s^{0} \cdot\left(\sigma^{\prime}(0)+\int_{\Gamma} \psi(0) n\right),
$$

for all $(f, g) \in L^{1}\left(0, T ; L^{2}(\Omega)\right) \times L^{1}\left(0, T ; \mathbb{R}^{2}\right)$, where $(\psi, \sigma)$ is the finite energy solution of equation $(10)$. We see that the above definition makes sense because the solution $(\psi, \sigma)$ of equation $(10)$ obeys $\psi(0) \in V$. This leads to the following estimate in the case $\phi^{0}=0$ :

$$
\|\phi\|_{C\left([0, T] ; L^{2}(\Omega)\right)}+\left\|\phi^{\prime}\right\|_{C\left([0, T] ; V^{\prime}\right)}+\|s\|_{C\left([0, T] ; \mathbb{R}^{2}\right)} \leq C\left(\left\|\phi^{1}\right\|_{V^{\prime}}+\left|s^{0}\right|+\left|s^{1}\right|\right)
$$

The estimate of $\|\phi\|_{C\left([0, T] ; L^{2}(\Omega)\right)}$ and $\|s\|_{C\left([0, T] ; \mathbb{R}^{2}\right)}$ can be obtained from (12) and from Theorem 2.1 applied to the finite energy solution $(\psi, \sigma)$ of equation (10). The estimate of $\left\|\phi^{\prime}\right\|_{C\left([0, T] ; V^{\prime}\right)}$ can be deduced from (12) and from (5) applied to the finite energy solution $(\psi, \sigma)$ of equation $(10)$, with $f=f_{1}^{\prime}, f_{1} \in C_{c}^{1}((0, T) ; V)$, and $g=0$. Taking $g=g_{1}^{\prime}, g_{1} \in C_{c}^{1}\left((0, T) ; \mathbb{R}^{2}\right)$, and $f=0$, from $(6)$ we can deduce an estimate on $\left\|\sigma^{\prime}\right\|_{L^{1}\left(0, T ; \mathbb{R}^{2}\right)}$, but not on $\left\|\sigma^{\prime}\right\|_{C\left([0, T] ; \mathbb{R}^{2}\right)}$. As a consequence, we cannot obtain an estimate of $\left\|s^{\prime}\right\|_{C\left([0, T] ; \mathbb{R}^{2}\right)}$ for the weak solution of equation (1) corresponding to $\left(\phi^{0}, \phi^{1}, s^{0}, s^{1}\right)=\left(0, \phi^{1}, s^{0}, s^{1}\right) \in\{0\} \times V^{\prime} \times \mathbb{R}^{2} \times \mathbb{R}^{2}$ and $u=0$. This is one of the effects of the coupling term $\int_{\Gamma} \phi^{\prime} n$.

The transposition method cannot be directly used to define weak solutions if $\phi^{0} \in L^{2}(\Omega)$ is arbitrary because the term $\int_{\Gamma} \phi^{0} n$ is not defined in this case and hence (11) cannot be used any longer (an adaptation of the transposition method to define weak solutions is given at the end of the section). To overcome this drawback we are going to define weak solutions by using the semigroup approach.

Let us recall that we have

$$
D(A)=D\left(A^{*}\right) \hookrightarrow Z
$$

with dense and continuous embedding. In this setting we identify $Z$ and $Z^{\prime}$. Thus we have

$$
D(A)=D\left(A^{*}\right) \hookrightarrow Z=Z^{\prime} \hookrightarrow\left(D\left(A^{*}\right)\right)^{\prime}=(D(A))^{\prime},
$$

with dense and continuous embeddings. Let $(S(t))_{t \in \mathbb{R}}$ be the strongly continuous group on $Z$ generated by $(A, D(A))$. When the initial condition of equation $(1)$ does not belong to $Z$, but only belongs to $\left(D\left(A^{*}\right)\right)^{\prime}$, 
we can still define weak solutions of equation (1) in $C\left([0, T] ; D\left(A^{*}\right)^{\prime}\right)$ by using the extrapolation method which extends the dynamics to a larger space (see [3], pp. 159-161). Introducing the unbounded operator $A_{1}$ on $D\left(A^{*}\right)$, with domain $D\left(A_{1}\right)=D\left(\left(A^{*}\right)^{2}\right)$, defined by $A_{1} z=A^{*} z$ for all $z \in D\left(\left(A^{*}\right)^{2}\right)$, we know that the adjoint of $A_{1}$ is the unbounded operator $A_{1}^{*}$ in $\left(D\left(A^{*}\right)\right)^{\prime}$, with domain $D\left(A_{1}^{*}\right)=Z$ (see [3]). We denote by $(\tilde{S}(t))_{t \in \mathbb{R}}$, the strongly continuous unitary group on $\left(D\left(A^{*}\right)\right)^{\prime}$ generated by $\left(A_{1}^{*}, Z\right)$. Then we have

$$
S(t)\left(\phi^{0}, \phi^{1}, s^{0}, s^{1}\right)=\tilde{S}(t)\left(\phi^{0}, \phi^{1}, s^{0}, s^{1}\right) \quad \text { for all } \quad\left(\phi^{0}, \phi^{1}, s^{0}, s^{1}\right) \in Z, \quad \text { and all } t \in \mathbb{R},
$$

and the following equality holds

$$
\left\|S(\cdot)\left(\phi^{0}, \phi^{1}, s^{0}, s^{1}\right)\right\|_{C\left([0, T] ;\left(D\left(A^{*}\right)\right)^{\prime}\right)}=\left\|\left(\phi^{0}, \phi^{1}, s^{0}, s^{1}\right)\right\|_{\left(D\left(A^{*}\right)\right)^{\prime}},
$$

for all $\left(\phi^{0}, \phi^{1}, s^{0}, s^{1}\right) \in Z$.

In order to exploit the fact that $(\tilde{S}(t))_{t \in \mathbb{R}}$ is a strongly continuous group on $\left(D\left(A^{*}\right)\right)^{\prime}$, we need a more precise characterization of $\left(D\left(A^{*}\right)\right)^{\prime}$. Observe that

$$
\left(\phi^{0}, \phi^{1}, s^{0}, s^{1}\right) \longmapsto\left(\left\|\phi^{0}\right\|_{L^{2}(\Omega)}+\left\|\phi^{1}\right\|_{V^{\prime}}+\left|s^{0}\right|+\left|s^{1}+\int_{\Gamma} \phi^{0} n\right|\right)
$$

is a norm on $Z$. Let $Y$ be the completion of $Z$ with respect to this norm, and denote this norm by $\|\cdot\|_{Y}$.

Theorem 3.1. The dual space $\left(D\left(A^{*}\right)\right)^{\prime}$ can be identified algebraically and topologically with $Y$.

Proof. Since $\left(D\left(A^{*}\right)\right)^{\prime}=(D(A))^{\prime}$, we are going to prove that $(D(A))^{\prime}$ can be identified algebraically and topologically with $Y$. Let us recall that the norm on $(D(A))^{\prime}$ is defined by

$$
\|w\|_{(D(A))^{\prime}}=\sup _{z \in D(A)} \frac{\left|(z, w)_{Z}\right|}{\|z\|_{D(A)}} .
$$

The graph norm on $D(A)$

$$
z \longmapsto\|z\|_{Z}+\|A z\|_{Z}
$$

is equivalent to the norm

$$
z=\left(\phi^{0}, \phi^{1}, s^{0}, s^{1}\right) \longmapsto\left\|\Delta \phi^{0}\right\|_{L^{2}(\Omega)}+\left\|\phi^{1}\right\|_{V}+\left|s^{0}\right|+\left|s^{1}\right| .
$$

For all $z=\left(\phi^{0}, \phi^{1}, s^{0}, s^{1}\right) \in D(A)$ and $w=\left(\psi^{0}, \psi^{1}, \sigma^{0}, \sigma^{1}\right) \in Z$, we have

$$
\left|(z, w)_{Z}\right|=\left|-\int_{\Omega} \Delta \phi^{0} \psi^{0}+\int_{\Omega} \phi^{1} \psi^{1}+s^{0} \cdot \sigma^{0}+s^{1} \cdot\left(\sigma^{1}+\int_{\Gamma} \psi^{0} n\right)\right| \leq C\|z\|_{D(A)}\|w\|_{Y} .
$$

Thus

$$
\|w\|_{(D(A))^{\prime}} \leq C\|w\|_{Y} \quad \text { for all } w \in Z .
$$

Let us prove the reverse inequality. Let $w=\left(\psi^{0}, \psi^{1}, \sigma^{0}, \sigma^{1}\right) \in Z$, and let $\phi^{0} \in H^{2}(\Omega)$ be the solution to the boundary value problem

$$
-\Delta \phi^{0}=\psi^{0} \quad \text { in } \Omega, \quad \phi^{0}=0 \quad \text { on } \Gamma_{e}, \quad \frac{\partial \phi^{0}}{\partial n}=\left(\sigma^{1}+\int_{\Gamma} \psi^{0} n\right) \cdot n \quad \text { on } \Gamma .
$$

Then $z=\left(\phi^{0}, 0,0, \sigma^{1}+\int_{\Gamma} \psi^{0} n\right) \in D(A)$ and

$$
\left|(z, w)_{Z}\right|=-\int_{\Omega} \Delta \phi^{0} \psi^{0}+\left|\sigma^{1}+\int_{\Gamma} \psi^{0} n\right|^{2}=\int_{\Omega}\left|\psi^{0}\right|^{2}+\left|\sigma^{1}+\int_{\Gamma} \psi^{0} n\right|^{2} .
$$


Since

it follows that

$$
\|w\|_{(D(A))^{\prime}} \geq \frac{\left|(z, w)_{Z}\right|}{\|z\|_{D(A)}}
$$

$$
\left\|\psi^{0}\right\|_{L^{2}(\Omega)}+\left|\sigma^{1}+\int_{\Gamma} \psi^{0} n\right| \leq C\|w\|_{(D(A))^{\prime}}
$$

Let $\phi^{1} \in V$ be such that

$$
\left\|\phi^{1}\right\|_{V}=1 \quad \text { and } \quad\left\langle\psi^{1}, \phi^{1}\right\rangle_{V^{\prime}, V}=\left\|\psi^{1}\right\|_{V^{\prime}} .
$$

Setting $z=\left(0, \phi^{1}, 0,0\right)$, we have

$$
\|w\|_{(D(A))^{\prime}} \geq \frac{\left|(z, w)_{Z}\right|}{\|z\|_{D(A)}}=\left\|\psi^{1}\right\|_{V^{\prime}} .
$$

Setting $z=\left(0,0, \sigma^{0}, 0\right)$, we have

$$
\|w\|_{(D(A))^{\prime}} \geq \frac{\left|(z, w)_{Z}\right|}{\|z\|_{D(A)}}=\left|\sigma^{0}\right| .
$$

Collecting together these different estimates, we obtain

$$
\|w\|_{Y} \leq C\|w\|_{(D(A))^{\prime}} \quad \text { for all } w \in Z .
$$

The theorem follows from (14), (15), and the fact that $Y$ is the closure of $Z$ with respect to the norm $\|\cdot\|_{Y}$, and $(D(A))^{\prime}$ is the closure of $Z$ with respect to the norm $\|\cdot\|_{(D(A))^{\prime}}$.

Remark 3.2. Due to Theorem $3.1,(\tilde{S}(t))_{t \in \mathbb{R}}$ is the strongly continuous group on $Y$ generated by the operator $\left(A_{1}^{*}, Z\right)$.

Theorem 3.3. Let $z_{0}=\left(\phi^{0}, \phi^{1}, s^{0}, s^{1}\right) \in Z$. The finite energy solution $S(\cdot) z_{0}=\left(\phi, \phi^{\prime}, s, s^{\prime}\right)$ to equation (1) with $u=0$ obeys the following estimate

$$
\begin{aligned}
\|\phi\|_{C\left([0, T] ; L^{2}(\Omega)\right)}+\left\|\phi^{\prime}\right\|_{C\left([0, T] ; V^{\prime}\right)}+\|s\|_{C\left([0, T] ; \mathbb{R}^{2}\right)}+\left\|s^{\prime}+\int_{\Gamma} \phi n\right\|_{C\left([0, T] ; \mathbb{R}^{2}\right)} & \leq C\left(\left\|\phi^{0}\right\|_{L^{2}(\Omega)}+\left\|\phi^{1}\right\|_{V^{\prime}}+\left|s^{0}\right|+\left|s^{1}+\int \phi_{\Gamma}^{0} n\right|\right) .
\end{aligned}
$$

Proof. The theorem is a direct consequence of the estimate

$$
\left\|S(\cdot) z_{0}\right\|_{C([0, T] ; Y)} \leq C\left\|z_{0}\right\|_{Y}
$$

Remark 3.4. We see that estimate (16) is better than the one obtained in (13).

Remark 3.5. It is clear from the definition of the space $Y$ that the combination $s^{\prime}+\int_{\Gamma} \phi n$ is a well-defined element of $C\left([0, T] ; \mathbb{R}^{2}\right)$ for weak solutions of equation (1) with $u=0$, given by the group $(\widetilde{S}(t))_{t \in \mathbb{R}}$ in the space $Y$ even though individual terms do not make sense. Further the Green formula (11) continues to hold good for a weak solution $(\phi, s)$ of $(3)$ and a finite energy solution $(\psi, \sigma)$ of $(10)$, if we accept to replace $s^{1}+\int_{\Gamma} \phi^{0} n$ by $\tilde{s}^{1}$ where $\left(\phi^{0}, \phi^{1}, s^{0}, \tilde{s}^{1}\right)=\tilde{J}\left(\phi^{0}, \phi^{1}, s^{0}, s^{1}\right)$, and the operator $\tilde{J}$ is the one introduced in Section 5 . This leads to the alternate definition given below.

Definition 3.6. Let $\left(\phi^{0}, \phi^{1}, s^{0}, s^{1}\right)$ belong to $Y$ and set $u=0$. A pair $(\phi, s) \in L^{\infty}\left(0, T ; L^{2}(\Omega)\right) \times L^{\infty}\left(0, T ; \mathbb{R}^{2}\right)$ is called a weak solution of equation (1) in the transposition sense if and only if

$$
\int_{Q} f \phi+\int_{0}^{T} g \cdot s=-\left(\phi^{0}, \psi^{\prime}(0)\right)_{L^{2}(\Omega)}+\left\langle\psi(0), \phi^{1}\right\rangle_{V, V^{\prime}}+\sigma(0) \cdot \tilde{s}^{1}-s^{0} \cdot\left(\sigma^{\prime}(0)+\int_{\Gamma} \psi(0) n\right)
$$


for all $(f, g) \in L^{1}\left(0, T ; L^{2}(\Omega)\right) \times L^{1}\left(0, T ; \mathbb{R}^{2}\right)$, where $\left(\phi^{0}, \phi^{1}, s^{0}, \tilde{s}^{1}\right)=\tilde{J}\left(\phi^{0}, \phi^{1}, s^{0}, s^{1}\right)$, and $(\psi, \sigma)$ is the finite energy solution of equation $(10)$.

We can easily check that if $\left(\phi^{0}, \phi^{1}, s^{0}, s^{1}\right)$ belongs to $Y$ and $u=0$, then equation (1) admits a unique solution in the sense of definition 3.6. This existence and uniqueness result is a consequence of Theorems 5.1 and 2.1 applied to the finite energy solution $(\psi, \sigma)$ of equation (10), and we have

$$
\|\phi\|_{C\left([0, T] ; L^{2}(\Omega)\right)}+\|s\|_{C\left([0, T] ; \mathbb{R}^{2}\right)} \leq C\left(\left\|\phi^{0}\right\|_{L^{2}(\Omega)}+\left\|\phi^{1}\right\|_{V^{\prime}}+\left|s^{0}\right|+\left|s^{1}+\int_{\Gamma} \phi^{0} n\right|\right) .
$$

However, there are difficulties in deducing estimates on $\phi^{\prime}$ and $s^{\prime}+\int_{\Gamma} \phi n$ directly from (17). Let us explain why in the following remark.

Remark 3.7. Because of the Green formula (11), it is clear that if $\left(\phi^{0}, \phi^{1}, s^{0}, s^{1}\right)$ belongs to $Z$ and $u=0$, the solution $(\phi, s)$ of equation (1) in the sense of definition 3.6 is such that $\left(\phi, \phi^{\prime}, s, s^{\prime}\right)=S(\cdot)\left(\phi^{0}, \phi^{1}, s^{0}, s^{1}\right)=$ $\tilde{S}(\cdot)\left(\phi^{0}, \phi^{1}, s^{0}, s^{1}\right)$. Let now $\left(\phi^{0}, \phi^{1}, s^{0}, s^{1}\right)$ belong to $Y$, and let $\left(\left(\phi_{k}^{0}, \phi_{k}^{1}, s_{k}^{0}, s_{k}^{1}\right)\right)_{k}$ be a sequence in $Z$, converging to $\left(\phi^{0}, \phi^{1}, s^{0}, s^{1}\right)$ in $Y$. Let $\left(\phi_{k}, s_{k}\right)$ be the solution of equation (1) corresponding to $\left(\phi_{k}^{0}, \phi_{k}^{1}, s_{k}^{0}, s_{k}^{1}\right)$ and to $u=0$. From estimate (16) it follows that $\left(\left(\phi_{k}, \phi_{k}^{\prime}, s_{k}, s_{k}^{\prime}+\int_{\Gamma} \phi_{k} n\right)\right)_{k}$ converges to $\tilde{J} \tilde{S}(\cdot)\left(\phi^{0}, \phi^{1}, s^{0}, s^{1}\right)$ in $L^{2}(\Omega) \times V^{\prime} \times \mathbb{R}^{2} \times \mathbb{R}^{2}$. From estimate (18), it follows that the sequence $\left(\left(\phi_{k}, s_{k}\right)\right)_{k}$ converges to the solution $(\phi, s)$ of equation (1) in the sense of definition 3.6. Setting $\left(\Phi, \Phi^{\prime}, \mathbf{s}, \tilde{\mathbf{s}}\right)=\tilde{J} \tilde{S}(\cdot)\left(\phi^{0}, \phi^{1}, s^{0}, s^{1}\right)$, we have $(\Phi, \mathbf{s})=(\phi, s)$, but we cannot deduce any estimate of $\left\|\phi_{k}^{\prime}\right\|_{C\left([0, T] ; V^{\prime}\right)}$ and $\left\|s_{k}^{\prime}+\int_{\Gamma} \phi_{k} n\right\|_{C\left([0, T] ; \mathbb{R}^{2}\right)}$ from the formulation (17).

Indeed, if we want to estimate $\left\|\phi^{\prime}\right\|_{C\left([0, T] ; V^{\prime}\right)}$ by the method used in $[10$, page 50-52], we have to consider the finite energy solution $(\psi, \sigma)$ of equation (10) with $f=f_{1}^{\prime}$ and $f_{1} \in C_{c}^{1}((0, T) ; V)$ and $g=0$. But Theorem 2.5 only provides an estimate of $\left\|\psi^{\prime}\right\|_{L^{1}\left(0, T ; L^{2}(\Omega)\right)}$ and not of $\left\|\psi^{\prime}\right\|_{L^{\infty}\left(0, T ; L^{2}(\Omega)\right)}$. This is the reason why estimate (16) cannot be directly deduced from the formulation (17). The same difficulty occurs in estimating $\left\|s^{\prime}+\int_{\Gamma} \phi n\right\|_{C\left([0, T] ; \mathbb{R}^{2}\right)}$. On the other hand estimate (16) is a direct consequence of the continuity on $Y$ of the group $(\tilde{S}(t))_{t \in \mathbb{R}}$.

An alternate way to prove estimate (16) may be to consider the finite energy solution $(\psi, \sigma)$ of

$$
\begin{array}{lll}
\psi^{\prime \prime}-\Delta \psi=0 & & \text { in } \quad Q, \\
\psi=0 & & \text { on } \Sigma_{e}, \\
\partial_{n} \psi=\sigma^{\prime} \cdot n & & \text { on } \Sigma, \\
\psi(\tau)=\psi_{\tau} \quad \text { and } & \psi^{\prime}(\tau)=0 & \text { in } \Omega, \\
\sigma^{\prime \prime}+\sigma=-\int_{\Gamma} \psi^{\prime} n & & \text { in } \quad(0, T), \\
\sigma(\tau)=\sigma_{\tau} \quad \text { and } & \sigma^{\prime}(\tau)=0 & \text { in } \quad \mathbb{R}^{2},
\end{array}
$$

and the variational equation

$\left\langle\psi_{\tau}, \phi^{\prime}(\tau)\right\rangle_{V, V^{\prime}}+\sigma_{\tau} \cdot\left(s^{\prime}(\tau)+\int_{\Gamma} \phi(\tau) n\right)=-\left(\phi^{0}, \psi^{\prime}(0)\right)_{L^{2}(\Omega)}+\left\langle\psi(0), \phi^{1}\right\rangle_{V, V^{\prime}}+\sigma(0) \cdot \tilde{s}^{1}-s^{0} \cdot\left(\sigma^{\prime}(0)+\int_{\Gamma} \psi(0) n\right)$,

where $\tau$ is arbitrarily fixed in $(0, T], \psi_{\tau} \in V, \sigma_{\tau} \in \mathbb{R}^{2}$, and $\left(\phi, \phi^{\prime}, s, s^{\prime}\right)$ is the finite energy solution of equation (1) with $u=0$. This method is used in [6] for another problem. As in [6], from (20) we can deduce that

$$
\left\|\phi^{\prime}(\tau)\right\|_{V^{\prime}}+\left\|s^{\prime}(\tau)+\int_{\Gamma} \phi(\tau) n\right\|_{\mathbb{R}^{2}} \leq C\left\|\left(\phi^{0}, \phi^{1}, s^{0}, s^{1}\right)\right\|_{Y},
$$


where $C$ is a constant independent of $\tau$. Next, taking the supremum with respect to $\tau$, we recover estimate (16). Thus we observe that, by this method, the continuity in $C([0, T] ; Y)$ is more complicated to prove than by directly using the semigroup property in the present case.

\section{INVERSE INEQUALITY}

The main results of this section are the inverse inequalities stated in Theorems 4.4 and 4.5. Both inequalities are stated for finite energy solutions even if the norm appearing in (24) is the norm of $Y$. Inequality (24) is used in Theorem 5.2 for finite energy solutions. In Section 5, the operator $\Lambda$ of the Hilbert Uniqueness Method is first defined in $Z$ and next extended to $Y$.

Consider the homogeneous problem:

$$
\begin{aligned}
& y^{\prime \prime}-\Delta y=0 \quad \text { in } Q, \\
& y=0 \quad \text { on } \Sigma_{e}, \\
& \partial_{n} y=r^{\prime} \cdot n \quad \text { on } \Sigma, \\
& y(0)=y^{0} \quad \text { and } \quad y^{\prime}(0)=y^{1} \quad \text { in } \Omega \text {, } \\
& r^{\prime \prime}+r=-\int_{\Gamma} y^{\prime} n \quad \text { in }(0, T), \\
& r(0)=r^{0} \quad \text { and } \quad r^{\prime}(0)=r^{1} \quad \text { in } \quad \mathbb{R}^{2} .
\end{aligned}
$$

Theorem 4.1. There exists a constant $C>0$ such that, for all $\left(y^{0}, y^{1}, r^{0}, r^{1}\right) \in Z$, the finite energy solution $(y, r)$ of equation (21) satisfies

$$
\left\|y^{\prime}(t)\right\|_{L^{2}(\Omega)}+\|y(t)\|_{V}+\left|r^{\prime}(t)\right|+|r(t)| \leq C\left(\int_{Q}\left(y^{\prime}\right)^{2}+\int_{0}^{T} r \cdot r\right)^{\frac{1}{2}}
$$

for all $0 \leq t \leq T$.

Proof. Let $\rho$ be the function defined by

With integration by parts we have

$$
\rho(t)=t^{2}(T-t)^{2}
$$

$$
\int_{Q} y^{\prime \prime} \rho y=-\int_{Q} \rho^{\prime} y y^{\prime}-\int_{Q} \rho\left(y^{\prime}\right)^{2}
$$

and

$$
\int_{Q}(-\Delta y) \rho y=\int_{Q} \rho|\nabla y|^{2}-\int_{\Sigma} \partial_{n} y \rho y=\int_{Q} \rho|\nabla y|^{2}-\int_{\Sigma} r^{\prime} \cdot n \rho y=\int_{Q} \rho|\nabla y|^{2}+\int_{0}^{T} \rho^{\prime} r \cdot \int_{\Gamma} y n+\int_{0}^{T} \rho r \cdot \int_{\Gamma} y^{\prime} n .
$$

On the other hand we have

$$
\int_{0}^{T} r^{\prime \prime} \cdot \rho r+\int_{0}^{T} \rho r \cdot r=-\int_{0}^{T} \rho r^{\prime} \cdot r^{\prime}-\int_{0}^{T} \rho^{\prime} r \cdot r^{\prime}+\int_{0}^{T} \rho r \cdot r=-\int_{0}^{T} \rho r \cdot \int_{\Gamma} y^{\prime} n
$$

Thus we have

$$
0=-\int_{Q} \rho^{\prime} y y^{\prime}-\int_{Q} \rho\left(y^{\prime}\right)^{2}+\int_{Q} \rho|\nabla y|^{2}+\int_{0}^{T} \rho^{\prime} r \cdot \int_{\Gamma} y n+\int_{0}^{T} \rho r^{\prime} \cdot r^{\prime}+\int_{0}^{T} \rho^{\prime} r \cdot r^{\prime}-\int_{0}^{T} \rho r \cdot r .
$$


Since $\rho^{\prime} \rho^{-1 / 2}$ belongs to $L^{\infty}(0, T)$, we see that, for all $\varepsilon>0$, there exists a constant $C(\varepsilon)>0$ such that

$$
\begin{gathered}
\left|\int_{Q} \rho^{\prime} y y^{\prime}\right| \leq \varepsilon \int_{Q} \rho y^{2}+C(\varepsilon) \int_{Q}\left(y^{\prime}\right)^{2}, \\
\left|\int_{0}^{T} \rho^{\prime} r \cdot \int_{\Gamma} y n\right| \leq \varepsilon \int_{0}^{T} \rho\left|\int_{\Gamma} y n\right|^{2}+C(\varepsilon) \int_{0}^{T} r \cdot r,
\end{gathered}
$$

and

$$
\left|\int_{0}^{T} \rho^{\prime} r \cdot r^{\prime}\right| \leq \varepsilon \int_{0}^{T} \rho r^{\prime} \cdot r^{\prime}+C(\varepsilon) \int_{0}^{T} r \cdot r
$$

We already know that $y(t) \in V$ for all $t \in[0, T]$. From the Poincaré inequality and a trace theorem it follows that

$$
\int_{Q} \rho|\nabla y|^{2} \geq \lambda_{0}\left(\int_{Q} \rho y^{2}+\int_{0}^{T} \rho\left|\int_{\Gamma} y n\right|^{2}\right),
$$

for some constant $\lambda_{0}>0$. Thus we can choose $\varepsilon>0$ small enough to have

$$
\int_{0}^{T} \rho(t)\left(\left\|y^{\prime}(t)\right\|_{L^{2}(\Omega)}^{2}+\|y(t)\|_{V}^{2}+\left|r^{\prime}(t)\right|^{2}+|r(t)|^{2}\right) \mathrm{d} t \leq C\left(\int_{Q}\left(y^{\prime}\right)^{2}+\int_{0}^{T} r \cdot r\right) .
$$

Since $E(0)=E(t)=\left\|y^{\prime}(t)\right\|_{L^{2}(\Omega)}^{2}+\|y(t)\|_{V}^{2}+\left|r^{\prime}(t)\right|^{2}+|r(t)|^{2}$, the proof is complete.

Theorem 4.2. There exists a constant $C>0$ such that the finite energy solution $(y, r)$ of equation (21) satisfies the following inverse inequality

$$
\left\|y^{0}\right\|_{L^{2}(\Omega)}+\left\|y^{1}\right\|_{V^{\prime}}+\left|r^{0}\right|+\left|r^{1}+\int_{\Gamma} y^{0} n\right| \leq C\left(\int_{Q} y^{2}+\int_{0}^{T} \zeta \cdot \zeta\right)^{\frac{1}{2}},
$$

for all $\left(y^{0}, y^{1}, r^{0}, r^{1}\right) \in V \times L^{2}(\Omega) \times \mathbb{R}^{2} \times \mathbb{R}^{2}$, where $\zeta$ is the solution to the differential equation

$$
\zeta^{\prime}(t)=r(t) \quad \text { in } \quad(0, T), \quad \text { and } \quad \zeta(0)=-r^{1}-\int_{\Gamma} y^{0} n
$$

Proof. Consider the boundary value problem

$$
\Delta \chi=y^{1} \quad \text { in } \quad \Omega, \quad \chi \in V, \quad \partial_{n} \chi=r^{0} \cdot n \quad \text { on } \quad \Gamma .
$$

The solution $\chi$ is equal to $\chi_{1}+\chi_{0}$, where $\chi_{1}$ is the solution of

$$
\Delta \chi_{1}=y^{1} \quad \text { in } \quad \Omega, \quad \chi_{1} \in V, \quad \partial_{n} \chi_{1}=0 \quad \text { on } \quad \Gamma,
$$

and $\chi_{0}$ is the solution of

$$
\Delta \chi_{0}=0 \quad \text { in } \quad \Omega, \quad \chi_{0} \in V, \quad \partial_{n} \chi_{0}=r^{0} \cdot n \quad \text { on } \quad \Gamma .
$$

We can easily verify that

and

$$
\left\|\chi_{1}\right\|_{V}=\left\|y^{1}\right\|_{V^{\prime}}
$$

Thus we have

$$
\left\|\chi_{0}\right\|_{V} \leq C_{1}\left|r^{0}\right|
$$

$$
\left\|y^{1}\right\|_{V^{\prime}}-C_{1}\left|r^{0}\right| \leq\|\chi\|_{V} .
$$


Let us set

and define the function $\zeta$ by

$$
w(x, t)=\int_{0}^{t} y(x, \tau) \mathrm{d} \tau+\chi(x)
$$

$$
\zeta(0)=-r^{1}-\int_{\Gamma} y^{0} n, \quad \zeta^{\prime}=r .
$$

The pair $(w, \zeta)$ obeys the system

$$
\begin{array}{ll}
w^{\prime \prime}-\Delta w=0 & \text { in } Q, \\
w=0 & \text { on } \Sigma_{e}, \\
\partial_{n} w=\zeta^{\prime} \cdot n & \text { on } \Sigma, \\
w(0)=\chi \quad \text { and } \quad w^{\prime}(0)=y^{0} & \text { in } \Omega, \\
\zeta^{\prime \prime}+\zeta=-\int_{\Gamma} w^{\prime} n & \text { in }(0, T), \\
\zeta(0)=-r^{1}-\int_{\Gamma} y^{0} n \quad \text { and } \quad \zeta^{\prime}(0)=r^{0} & \text { in } \mathbb{R}^{2} .
\end{array}
$$

Applying Theorem 4.1 to $(w, \zeta)$ we get

$$
\|\chi\|_{V}+\left\|y^{0}\right\|_{L^{2}(\Omega)}+\left|r^{1}+\int_{\Gamma} y^{0} n\right|+\left|r^{0}\right| \leq C\left(\int_{Q} y^{2}+\int_{0}^{T} \zeta \cdot \zeta\right)^{\frac{1}{2}}
$$

With estimate (22), we finally have

$$
\begin{aligned}
& \left\|y^{1}\right\|_{V^{\prime}}+\left\|y^{0}\right\|_{L^{2}(\Omega)}+\left|r^{1}+\int_{\Gamma} y^{0} n\right|+\left|r^{0}\right| \\
& \quad \leq\|\chi\|_{V}+\left\|y^{0}\right\|_{L^{2}(\Omega)}+\left|r^{1}+\int_{\Gamma} y^{0} n\right|+\left(1+C_{1}\right)\left|r^{0}\right| \leq C\left(\int_{Q} y^{2}+\int_{0}^{T} \zeta \cdot \zeta\right)^{\frac{1}{2}}
\end{aligned}
$$

and the proof is complete.

We can now eliminate the term $\int_{0}^{T} r \cdot r$ from the right hand side in Theorem 4.1.

Theorem 4.3. There exists a constant $C>0$ such that, for every $y^{0} \in V$, every $y^{1} \in L^{2}(\Omega)$, every $r^{0} \in \mathbb{R}^{2}$, and every $r^{1} \in \mathbb{R}^{2}$, the finite energy solution $(y, r)$ of equation (21) satisfies

$$
\|r\|_{L^{\infty}\left(0, T ; \mathbb{R}^{2}\right)} \leq C\left\|y^{\prime}\right\|_{L^{2}(Q)}
$$

Proof. We argue by contradiction. We suppose that there exist a sequence $\left(y_{j}, r_{j}\right)_{j}$ of solutions of equation (21) such that $\left\|r_{j}\right\|_{L^{\infty}\left(0, T ; \mathbb{R}^{2}\right)}=1$ and $\lim _{j \rightarrow \infty} \int_{Q}\left(y_{j}^{\prime}\right)^{2}=0$. Using the inverse inequality of Theorem 4.1 we can suppose that there exist $y$ and $r$ such that

$$
y_{j} \stackrel{*}{\rightarrow} y \quad \text { in } \quad L^{\infty}(0, T ; V), \quad y_{j}^{\prime} \stackrel{*}{\rightarrow} 0 \quad \text { in } \quad L^{\infty}\left(0, T ; L^{2}(\Omega)\right), \quad y_{j}^{\prime} \rightarrow 0 \quad \text { in } \quad L^{2}\left(0, T ; L^{2}(\Omega)\right)
$$

and

$$
r_{j} \stackrel{*}{\rightarrow} r \quad \text { in } \quad L^{\infty}\left(0, T ; \mathbb{R}^{2}\right), \quad r_{j}^{\prime} \stackrel{*}{\rightarrow} r^{\prime} \quad \text { in } \quad L^{\infty}\left(0, T ; \mathbb{R}^{2}\right)
$$


By Arzela-Ascoli Theorem it follows that the sequence $\left(r_{j}\right)_{j}$ tends to $r$ in $C\left([0, T] ; \mathbb{R}^{2}\right)$. Observe that $y^{\prime}=0$. On the other hand $\partial_{n} y=r^{\prime} \cdot n$. Thus

$$
\partial_{n} y^{\prime}=r^{\prime \prime} \cdot n=0 \quad \text { on } \quad \Sigma .
$$

Therefore $r^{\prime \prime}=0$ in $(0, T)$.

Let $\sigma \in C^{1}\left([0, T] ; \mathbb{R}^{2}\right)$ be such that $\sigma(0)=\sigma(T)=0$. Multiplying by $\sigma$ the equation satisfied by $r_{j}$, we have:

$$
\int_{0}^{T}\left(-r_{j}^{\prime} \sigma^{\prime}+r_{j} \sigma\right)=\int_{0}^{T} \sigma^{\prime} \int_{\Gamma} y_{j} \cdot n .
$$

Passing to the limit, we obtain

$$
\int_{0}^{T}\left(-r^{\prime} \sigma^{\prime}+r \sigma\right)=\int_{\Gamma}\left(\left(\int_{0}^{T} \sigma^{\prime}\right) \cdot n y\right)=0 .
$$

Thus

$$
r^{\prime \prime}+r=0 \quad \text { in the sense of distributions in } \quad(0, T) .
$$

Since $r^{\prime \prime}=0$, we have $r=0$. This is clearly in contradiction with $\left\|r_{j}\right\|_{L^{\infty}\left(0, T ; \mathbb{R}^{2}\right)}=1$ and $\left(r_{j}\right)_{j}$ tends to $r$ in $C\left([0, T] ; \mathbb{R}^{2}\right)$.

Theorem 4.4. There exists a constant $C>0$ such that the finite energy solution $(y, r)$ of equation (21) satisfies the following inverse inequality

$$
\left\|y^{0}\right\|_{L^{2}(\Omega)}+\left\|y^{1}\right\|_{V^{\prime}}+\left|r^{0}\right|+\left|r^{1}+\int_{\Gamma} y^{0} n\right| \leq C\|y\|_{L^{2}(Q)}, \quad \text { for all }\left(y^{0}, y^{1}, r^{0}, r^{1}\right) \in Z .
$$

Proof. We use the notation in the proof of Theorem 4.2. Applying Theorem 4.3 to the solution $(w, \zeta)$ of equation (23), we have

$$
\|\zeta\|_{L^{\infty}\left(0, T ; \mathbb{R}^{2}\right)} \leq C\left\|w^{\prime}\right\|_{L^{2}(Q)}=C\|y\|_{L^{2}(Q)} .
$$

The proof follows from the inverse inequality stated in Theorem 4.2.

Theorem 4.5. Suppose that $\left(y^{0}, y^{1}, r^{0}, r^{1}\right) \in Z$. The finite energy solution (y,r) of equation (21) satisfies the following inverse inequality

$$
\left\|y^{0}\right\|_{V}+\left\|y^{1}\right\|_{L^{2}(\Omega)}+\left|r^{0}\right|+\left|r^{1}\right| \leq C\left\|y^{\prime}\right\|_{L^{2}(Q)} .
$$

Proof. The result is a direct consequence of Theorems 4.1 and 4.3 .

\section{Proof of Theorem 1.1}

Set $F=L^{2}(\Omega) \times V^{\prime} \times \mathbb{R}^{2} \times \mathbb{R}^{2}$ and $F^{\prime}=L^{2}(\Omega) \times V \times \mathbb{R}^{2} \times \mathbb{R}^{2}$. Define the operator $J$ from $Z$ into $F$ by

$$
J\left(\phi^{0}, \phi^{1}, s^{0}, s^{1}\right)=\left(\phi^{0}, \phi^{1}, s^{0}, s^{1}+\int_{\Gamma} \phi^{0} n\right) .
$$

Notice that $\|J(z)\|_{F}=\|z\|_{Y}$ for all $z \in Z$. By density and continuity, $J$ can be uniquely extended to a bounded linear operator $\widetilde{J}$ from $Y$ to $F$ such that $\|\widetilde{J}(z)\|_{F}=\|z\|_{Y}$ for all $z \in Y$.

Theorem 5.1. The operator $\widetilde{J}$ is an isomorphism from $Y$ to $F$.

Proof. First prove that the operator $\widetilde{J}$ is surjective from $Y$ to $F$. Since $Y$ is complete, $\tilde{J}(Y)$ is closed in $F$. Therefore, it is sufficient to show that $\widetilde{J}(Y)$ or $J(Z)$ is dense in $F$. But $J(Z)=Z$, thus $\tilde{J}$ is surjective. Since $\widetilde{J}$ is an isometry, $\widetilde{J}$ is an isomorphism from $Y$ to $F$. 
Theorem 5.2. Let $P \in \mathcal{L}\left(Z ; L^{2}(\Omega)\right)$ be defined by $\left(\phi^{0}, \phi^{1}, s^{0}, s^{1}\right) \mapsto \phi^{0}$. The mapping

$$
(z, w) \longmapsto \int_{0}^{T}(P S(t) z, P S(t) w)_{L^{2}(\Omega)} \mathrm{d} t
$$

defines an inner product on $Z$, denoted by $(\cdot, \cdot)_{Y}$, whose norm is equivalent to $\|\cdot\|_{Y}$.

Proof. The proof follows from Theorems 3.3 and 4.4 .

Proof of Theorem 1.1. Consider the mapping from $Z$ into $F^{\prime}$ defined by

$$
\Lambda:\left(y^{0}, y^{1}, r^{0}, r^{1}\right) \longmapsto\left(-\psi^{\prime}(0), \psi(0),-\sigma^{\prime}(0)-\int_{\Gamma} \psi(0) n, \sigma(0)\right),
$$

where $(\psi, \sigma)$ is the finite energy solution to the equation

$$
\begin{aligned}
& \psi^{\prime \prime}-\Delta \psi=y \quad \text { in } Q, \\
& \psi=0 \quad \text { on } \Sigma_{e} \text {, } \\
& \partial_{n} \psi=\sigma^{\prime} \cdot n \quad \text { on } \Sigma \text {, } \\
& \psi(T)=0 \quad \text { and } \quad \psi^{\prime}(T)=0 \quad \text { in } \Omega \text {, } \\
& \sigma^{\prime \prime}+\sigma=-\int_{\Gamma} \psi^{\prime} n \quad \text { in }(0, T) \text {, } \\
& \sigma(T)=0 \quad \text { and } \quad \sigma^{\prime}(T)=0 \quad \text { in } \quad \mathbb{R}^{2} \text {, }
\end{aligned}
$$

and $(y, r)$ is the finite energy solution to equation (21) corresponding to $\left(y^{0}, y^{1}, r^{0}, r^{1}\right)$. Let $\left(\phi^{0}, \phi^{1}, s^{0}, s^{1}\right) \in Z$ and denote by $(\phi, s)$ the solution to equation (1) with $u=0$. Applying formula (11), we obtain

$$
\left\langle\Lambda\left(y^{0}, y^{1}, r^{0}, r^{1}\right), J\left(\phi^{0}, \phi^{1}, s^{0}, s^{1}\right)\right\rangle_{F^{\prime}, F}=\int_{Q} y \phi .
$$

From Theorems 5.1 and 5.2 it follows that

$$
\begin{aligned}
\left\langle\Lambda\left(y^{0}, y^{1}, r^{0}, r^{1}\right), J\left(\phi^{0}, \phi^{1}, s^{0}, s^{1}\right)\right\rangle_{F^{\prime}, F} & =\left(\left(y^{0}, y^{1}, r^{0}, r^{1}\right),\left(\phi^{0}, \phi^{1}, s^{0}, s^{1}\right)\right)_{Y} \\
& \leq C\left\|\left(y^{0}, y^{1}, r^{0}, r^{1}\right)\right\|_{Y}\left\|\left(\phi^{0}, \phi^{1}, s^{0}, s^{1}\right)\right\|_{Y}=C\left\|\left(y^{0}, y^{1}, r^{0}, r^{1}\right)\right\|_{Y}\left\|J\left(\phi^{0}, \phi^{1}, s^{0}, s^{1}\right)\right\|_{F}
\end{aligned}
$$

Thus $\Lambda$ is continuous from $\left(Z,\|\cdot\|_{Y}\right)$ into $F^{\prime}$. By density and continuity, $\Lambda$ can be uniquely extended to a bounded linear operator $\widetilde{\Lambda}$ from $Y$ to $F^{\prime}$. Equation (26) can be rewritten in the form

$$
\left\langle\widetilde{J}^{*} \widetilde{\Lambda}\left(y^{0}, y^{1}, r^{0}, r^{1}\right),\left(\phi^{0}, \phi^{1}, s^{0}, s^{1}\right)\right\rangle_{Y^{\prime}, Y}=\left(\left(y^{0}, y^{1}, r^{0}, r^{1}\right),\left(\phi^{0}, \phi^{1}, s^{0}, s^{1}\right)\right)_{Y}
$$

for all $\left(y^{0}, y^{1}, r^{0}, r^{1}\right) \in Y$, and all $\left(\phi^{0}, \phi^{1}, s^{0}, s^{1}\right) \in Y$.

Equation (28) implies that $\widetilde{J}^{*} \widetilde{\Lambda}$ is an isomorphism from $Y$ onto $Y^{\prime}$. Since $\widetilde{J}^{*}$ is an isomorphism from $Y$ onto $F$, it follows that $\widetilde{\Lambda}=\left(\widetilde{J}^{*}\right)^{-1}\left(\widetilde{J}^{*} \widetilde{\Lambda}\right)$ is an isomorphism from $Y$ onto $F^{\prime}$.

Let us start with $\left(\phi^{0}, \phi^{1}, s^{0}, s^{1}\right)$ in $Z$. Notice that $\left(-\phi^{1}, \phi^{0},-s^{1}-\int_{\Gamma} \phi^{0} n, s^{0}\right)$ belongs to $F^{\prime}$. Let $\left(y^{0}, y^{1}, r^{0}, r^{1}\right) \in$ $Y$ be the solution to the equation

$$
\widetilde{\Lambda}\left(y^{0}, y^{1}, r^{0}, r^{1}\right)=\left(-\phi^{1}, \phi^{0},-s^{1}-\int_{\Gamma} \phi^{0} n, s^{0}\right) .
$$


Denote by $(y, r)$ the solution of equation (21) corresponding to $\left(y^{0}, y^{1}, r^{0}, r^{1}\right)$, and by $(\psi, \sigma)$ the solution to equation (25) corresponding to $y$. From the definition of $\widetilde{\Lambda}$ it follows that

$$
\psi(0)=\phi^{0}, \quad \psi^{\prime}(0)=\phi^{1}, \quad \sigma(0)=s^{0}, \quad \sigma^{\prime}(0)=s^{1} .
$$

Therefore if we take $u=y$ the solution $(\phi, s)$ to equation (1) obeys $\phi(T)=\phi^{\prime}(T)=0$ and $s(T)=s^{\prime}(T)=0$. Theorem 1.1 is proved.

\section{Controllability of Weak solutions}

The main result of the two following sections is a controllabity result for weak solutions stated below.

Theorem 6.1. For all $\left(\phi^{0}, \phi^{1}, s^{0}, s^{1}\right) \in Y$, there exists a control $u \in\left(H^{1}\left(0, T ; L^{2}(\Omega)\right)\right)^{\prime}$ such that the weak solution to equation (1) obeys

$$
\phi(T)=\phi^{\prime}(T)=0 \quad \text { and } \quad s(T)=s^{\prime}(T)=0 .
$$

Throughout this section, we fix $\left(y^{0}, y^{1}, r^{0}, r^{1}\right) \in Z$ and consider the corresponding finite energy solution $(y, r)$ satisfying (21). All estimates will be in terms of $y$.

To prove Theorem 6.1 , we need to consider the system (31) wherein we take $f=-d_{t}\left[y^{\prime}\right]$. This new object $-d_{t}\left[y^{\prime}\right]$ is associated with $y$. To define it, we recall that $(y, r)$ belongs to $\left(C([0, T] ; V) \cap C^{1}\left([0, T] ; L^{2}(\Omega)\right) \cap\right.$ $\left.H^{2}\left(0, T ; V^{\prime}\right)\right) \times C^{1}\left([0, T] ; \mathbb{R}^{2}\right)$. Thus we can define $-d_{t}\left[y^{\prime}\right]$ as an element of $\left(H^{1}\left(0, T ; L^{2}(\Omega)\right)\right)^{\prime}$ by

$$
\left\langle-d_{t}\left[y^{\prime}\right], \theta\right\rangle_{\left(H^{1}\left(0, T ; L^{2}(\Omega)\right)\right)^{\prime}, H^{1}\left(0, T ; L^{2}(\Omega)\right)}=\int_{Q} y^{\prime}(x, t) \theta^{\prime}(x, t) \mathrm{d} x \mathrm{~d} t,
$$

for all $\theta \in H^{1}\left(0, T ; L^{2}(\Omega)\right)$.

With the above definition, we consider the following system

$$
\begin{array}{lll}
\psi^{\prime \prime}-\Delta \psi=f & \text { in } Q, \\
\psi=0 & & \text { on } \Sigma_{e}, \\
\partial_{n} \psi=\sigma^{\prime} \cdot n & \text { on } \Sigma, \\
\psi(T)=0 \quad \text { and } & \psi^{\prime}(T)=0 & \text { in } \Omega, \\
\sigma^{\prime \prime}+\sigma=-\int_{\Gamma} \psi^{\prime} n & & \text { in }(0, T), \\
\sigma(T)=0 \quad \text { and } & \sigma^{\prime}(T)=0 & \text { in } \mathbb{R}^{2} .
\end{array}
$$

Since $-d_{t}\left[y^{\prime}\right]$ does not belong to $L^{1}\left(0, T ; V^{\prime}\right)$, equation (31) with $f=-d_{t}\left[y^{\prime}\right]$ cannot be studied in the framework of weak solutions introduced in section 3 . We need another one which is provided in this section. The proof of Theorem 6.1 which uses (31) is given in Section 7. We can define the solutions of (31) by the transposition method (see Def. 6.8 below). By using the same method, we can prove that equation (31) admits a unique solution $(\psi, \sigma)$ in $L^{\infty}\left(0, T ; L^{2}(\Omega)\right) \times L^{\infty}\left(0, T ; \mathbb{R}^{2}\right)$ (Th. 6.9). This solution belongs to $C\left([0, T] ; L^{2}(\Omega)\right) \times C\left([0, T] ; \mathbb{R}^{2}\right)$, but $\psi^{\prime}$ does not belong to $C\left([0, T] ; V^{\prime}\right)$ and consequently, at this stage, we are not able to state a Green formula. But, as observed below, $-d_{t}\left[y^{\prime}\right]$ can be identified with a vector valued measure belonging to $L^{2}\left(0, T ; V^{\prime}\right)+$ $\mathcal{M}\left([0, T] ; L^{2}(\Omega)\right)$ and this enables us to prove that $\psi^{\prime}$ belongs to $L^{\infty}\left(0, T ; V^{\prime}\right)$ and $\sigma^{\prime}$ belongs to $L^{\infty}\left(0, T ; \mathbb{R}^{2}\right)$ and that there exists a unique $\left(\psi^{0}, \psi^{1}, \sigma^{0}, \tilde{\sigma}^{1}\right)$ in $L^{2}(\Omega) \times V^{\prime} \times \mathbb{R}^{2} \times \mathbb{R}^{2}$ which satisfies some Green formula (see Th. 6.9). In the case where the solution $(\psi, \sigma)$ is regular, the quadruplet $\left(\psi^{0}, \psi^{1}, \sigma^{0}, \tilde{\sigma}^{1}\right)$ is equal to $\left(\psi(0), \psi^{\prime}(0), \sigma(0), \sigma^{\prime}(0)+\int_{\Gamma} \psi(0) n\right)$. 
To see that $-d_{t}\left[y^{\prime}\right]$ is a vector valued measure, belonging to $L^{2}\left(0, T ; V^{\prime}\right)+\mathcal{M}\left([0, T] ; L^{2}(\Omega)\right)$, we observe that

$$
\begin{aligned}
\left\langle-d_{t}\left[y^{\prime}\right], \theta\right\rangle_{\left(L^{2}\left(0, T ; V^{\prime}\right)+\mathcal{M}\left([0, T] ; L^{2}(\Omega)\right)\right),} & \left(L^{2}(0, T ; V) \cap C\left([0, T] ; L^{2}(\Omega)\right)\right) \\
& =\int_{0}^{T}\left\langle-y^{\prime \prime}(t), \theta(t)\right\rangle_{V^{\prime}, V} \mathrm{~d} t+\left(y^{\prime}(T), \theta(T)\right)_{L^{2}(\Omega)}-\left(y^{\prime}(0), \theta(0)\right)_{L^{2}(\Omega)}
\end{aligned}
$$

for all $\theta \in\left(L^{2}(0, T ; V) \cap C\left([0, T] ; L^{2}(\Omega)\right)\right.$. This definition for $-d_{t}\left[y^{\prime}\right]$ is possible because $-y^{\prime \prime}$ belongs to $L^{2}\left(0, T ; V^{\prime}\right)$. Since

$$
\int_{0}^{T}\left\langle-y^{\prime \prime}(t), \theta(t)\right\rangle_{V^{\prime}, V} \mathrm{~d} t+\left(y^{\prime}(T), \theta(T)\right)_{L^{2}(\Omega)}-\left(y^{\prime}(0), \theta(0)\right)_{L^{2}(\Omega)}=\int_{0}^{T}\left(y^{\prime}(t), \theta^{\prime}(t)\right)_{L^{2}(\Omega)},
$$

for all $\theta \in\left(L^{2}(0, T ; V) \cap C\left([0, T] ; L^{2}(\Omega)\right)\right) \cap H^{1}\left(0, T ; L^{2}(\Omega)\right)$, it is clear that

$$
-d_{t}\left[y^{\prime}\right] \in\left(L^{2}\left(0, T ; V^{\prime}\right)+\mathcal{M}\left([0, T] ; L^{2}(\Omega)\right)\right) \cap\left(H^{1}\left(0, T ; L^{2}(\Omega)\right)\right)^{\prime} .
$$

Formula (32) means that $-d_{t}\left[y^{\prime}\right]=-y^{\prime \prime}+y^{\prime}(T) \otimes \delta_{T}-y^{\prime}(0) \otimes \delta_{0}$ and accordingly, we can decompose (31) into two parts. Due to the definition of the space $Y$, the source term $-y^{\prime \prime} \in L^{2}\left(0, T ; V^{\prime}\right)$ can be directly handled in the framework of weak solutions of Section 3. The measure $\left(y^{\prime}(T) \otimes \delta_{T}-y^{\prime}(0) \otimes \delta_{0}\right) \in \mathcal{M}\left([0, T] ; L^{2}(\Omega)\right)$ can be approximated by sequences in $L^{1}\left(0, T ; L^{2}(\Omega)\right)$ and the required estimates for $(\psi, \sigma)$ can be obtained by a passage to the limit (see the proof of Th. 6.7). After these preliminary remarks, let us now turn to formal definitions and proofs.

Following the decomposition $-d_{t}\left[y^{\prime}\right]=-y^{\prime \prime}+\mu$ with $\mu=y^{\prime}(T) \otimes \delta_{T}-y^{\prime}(0) \otimes \delta_{0}$, we split the solution of (31) into two parts:

$$
(\psi, \sigma)=\left(\psi_{1}, \sigma_{1}\right)+\left(\psi_{2}, \sigma_{2}\right)
$$

where $\left(\psi_{1}, \sigma_{1}\right)$ is the weak solution of $(31)$ with the source term $\left(-y^{\prime \prime}\right)$ and $\left(\psi_{2}, \sigma_{2}\right)$ corresponds to the source term $\mu$. Our first result is concerned with $\left(\psi_{1}, \sigma_{1}\right)$.

Definition 6.2. A function $(\psi, \sigma) \in L^{\infty}\left(0, T ; L^{2}(\Omega)\right) \times L^{\infty}\left(0, T ; \mathbb{R}^{2}\right)$ is a weak solution to equation (31) with the source term $f=-y^{\prime \prime}$ if

$$
\int_{Q} \psi u+\int_{0}^{T} \sigma \cdot v=-\int_{0}^{T}\left\langle\phi(t), y^{\prime \prime}(t)\right\rangle_{V, V^{\prime}} \mathrm{d} t
$$

for all $u \in L^{1}\left(0, T ; L^{2}(\Omega)\right), v \in L^{1}\left(0, T ; \mathbb{R}^{2}\right)$, where $(\phi, s)$ is the finite energy solution to equation (3) corresponding to $\left(\phi^{0}, \phi^{1}, s^{0}, s^{1}\right)=0$.

Theorem 6.3. There is a unique weak solution to (31) with the source term $f=-y^{\prime \prime}$ and it admits the following estimates:

$$
\begin{gathered}
\left\|\psi_{1}\right\|_{L^{\infty}\left(0, T ; L^{2}(\Omega)\right)}+\left\|\psi_{1}^{\prime}\right\|_{L^{\infty}\left(0, T ; V^{\prime}\right)}+\left\|\sigma_{1}\right\|_{L^{\infty}\left(0, T ; \mathbb{R}^{2}\right)}+\left\|\sigma_{1}^{\prime}\right\|_{L^{\infty}\left(0, T ; \mathbb{R}^{2}\right)} \leq C\left\|y^{\prime}\right\|_{C\left([0, T] ; L^{2}(\Omega)\right)}, \\
\left\|\sigma_{1}^{\prime}+\int_{\Gamma} \psi_{1} n\right\|_{L^{\infty}\left(0, T ; \mathbb{R}^{2}\right)} \leq C\left\|y^{\prime}\right\|_{C\left([0, T] ; L^{2}(\Omega)\right)} .
\end{gathered}
$$

Further, it enjoys the regularity property

$$
\left(\psi_{1}, \psi_{1}^{\prime}, \sigma_{1}, \sigma_{1}^{\prime}+\int_{\Gamma} \psi_{1} n\right) \in C([0, T] ; F) \quad \text { and } \quad\left(\psi_{1}, \psi_{1}^{\prime}, \sigma_{1}, \sigma_{1}^{\prime}\right) \in C([0, T] ; F) .
$$


Moreover the following formula holds

$$
\begin{aligned}
& \int_{Q} \psi_{1} u+\int_{0}^{T} \sigma_{1} \cdot v=\int_{Q} y^{\prime} \phi^{\prime}-\left(y^{\prime}(T), \phi(T)\right)_{L^{2}(\Omega)} \\
&-\left(\phi^{1}, \psi_{1}(0)\right)_{L^{2}(\Omega)}+\left\langle\phi^{0}, \psi_{1}^{\prime}(0)\right\rangle_{V, V^{\prime}}+s^{0} \cdot\left(\sigma_{1}^{\prime}(0)+\int_{\Gamma} \psi_{1}(0) n\right)-\left(s^{1}+\int_{\Gamma} \phi^{0} n\right) \cdot \sigma_{1}(0)
\end{aligned}
$$

for all $u \in L^{1}\left(0, T ; L^{2}(\Omega)\right), v \in L^{1}\left(0, T ; \mathbb{R}^{2}\right)$, and $\left(\phi^{0}, \phi^{1}, s^{0}, s^{1}\right) \in Z$, where $(\phi, s)$ is the finite energy solution to equation (3). (In formula (33), $\left(\psi_{1}(0), \psi_{1}^{\prime}(0), \sigma_{1}(0),\left(\sigma_{1}^{\prime}(0)+\int_{\Gamma} \psi_{1}(0) n\right)\right)$, is well defined in $F$ because $\left(\psi_{1}, \psi_{1}^{\prime}, \sigma_{1}, \sigma_{1}^{\prime}+\int_{\Gamma} \psi_{1} n\right) \in C([0, T] ; F)$.)

Proof. First of all, it is clear that the weak solution is unique if it exists. To prove the stated estimates, we proceed in steps.

Step 1. Since $y^{\prime \prime} \in C\left([0, T] ; V^{\prime}\right)$ (see Th. 3.1), a solution $\left(\psi_{1}, \sigma_{1}\right)$ can be obtained via the evolution group $(\widetilde{S}(t))_{t \geq 0}$ on the space $Y$.

To check that it is also a weak solution in the sense of the Definition 7.1, we consider a finite energy solution $(\phi, s)$ to the system $(3)$ with $\left(\phi^{0}, \phi^{1}, s^{0}, s^{1}\right)=0$. According to our remarks in Section 3 , it is perfectly legal to do integration by parts between $(\phi, s)$ and $\left(\psi_{1}, \sigma_{1}\right)$ and the corresponding Green formula can be written as

$$
\int_{Q} \psi_{1} u+\int_{0}^{T} \sigma_{1} \cdot v=-\int_{0}^{T}\left\langle\phi(t), y^{\prime \prime}(t)\right\rangle_{V, V^{\prime}}
$$

Thus the semigroup solution is also the solution in the sense of transposition stated in Definition 6.2. The right hand side can be further integrated to yield

$$
\int_{Q} \psi_{1} u+\int_{0}^{T} \sigma_{1} \cdot v=\int_{Q} \phi^{\prime} y^{\prime}-\left(y^{\prime}(T), \phi(T)\right)_{L^{2}(\Omega)} .
$$

Step 2. To obtain the second set of estimates, using Theorem 2.1 we majorize the right hand side of the above relation by

$$
\left\|y^{\prime}\right\|_{L^{\infty}\left(0, T, L^{2}(\Omega)\right)}\left(\left\|\phi^{\prime}\right\|_{L^{1}\left(0, T, L^{2}(\Omega)\right)}+\|\phi\|_{L^{\infty}\left(0, T ; L^{2}(\Omega)\right)}\right) \leq C\left\|y^{\prime}\right\|_{L^{\infty}\left(0, T ; L^{2}(\Omega)\right)}\left(\|u\|_{L^{1}\left(0, T, L^{2}(\Omega)\right)}+\|v\|_{L^{1}\left(0, T ; \mathbb{R}^{2}\right)}\right) .
$$

Since $(u, v)$ are arbitrary, the stated estimate on $\left(\psi_{1}, \sigma_{1}\right)$ follows.

Estimate on $\left(\psi_{1}^{\prime}, \sigma_{1}^{\prime}\right)$. We make the choice $u=f_{1}^{\prime}$ with $f_{1} \in C_{c}^{\prime}((0, T) ; V)$ and $v=v_{1}^{\prime}$ with $v_{1} \in C_{c}^{1}\left((0, T) ; \mathbb{R}^{2}\right)$. Above relation then becomes

$$
\int_{Q} \psi_{1} f_{1}^{\prime}+\int_{0}^{T} \sigma_{1} \cdot v_{1}^{\prime}=\int_{Q} y^{\prime} \phi^{\prime}-\left(y^{\prime}(T), \phi(T)\right)_{L^{2}(\Omega)} .
$$

This time around, using Theorem 2.5, we majorize the right side by

$$
C\left\|y^{\prime}\right\|_{L^{\infty}\left(0, T ; L^{2}(\Omega)\right)}\left(\left\|f_{1}\right\|_{L^{1}(0, T ; V)}+\left\|v_{1}\right\|_{L^{1}\left(0, T ; \mathbb{R}^{2}\right)}\right)
$$

Since $\left(f_{1}, v_{1}\right)$ are arbitrary, we obtain the required estimate on $\left(\psi_{1}^{\prime}, \sigma_{1}^{\prime}\right)$.

Step 3. The method presented in the above step seems to be inadequate to prove the last inequality. We present now a different argument to achieve this.

We consider a finite energy solution $(\phi, s)$ to $(3)$ with $u=0, v=0, \phi^{0}=\phi^{1}=0, s^{1}=0$ but $s^{0} \in \mathbb{R}^{2}$ arbitrary. 
Fixing $t_{0}$ such that $0 \leq t_{0}<T$, the new idea consists of introducing

$$
\left(\phi_{t_{0}}, s_{t_{0}}\right)(\tau)=(\phi, s)\left(\tau-t_{0}\right) \text { for } t_{0} \leq \tau \leq T
$$

Since $(\phi, s)$ is a solution to $(3)$ on $[0, T] \times \Omega$, it is clear that $\left(\phi_{t_{0}}, s_{t_{0}}\right)$ satisfies $(3)$ on $\left[t_{0}, T\right] \times \Omega$. Integration by parts between $\left(\psi_{1}, \sigma_{1}\right)$ and $\left(\phi_{t_{0}}, s_{t_{0}}\right)$ gives

$$
s^{0} \cdot\left(\sigma_{1}^{\prime}\left(t_{0}\right)+\int_{\Gamma} \psi_{1}\left(t_{0}\right) n\right)=-\int_{t_{0}}^{T} y^{\prime} \phi_{t_{0}}^{\prime}+\left(y^{\prime}(T), \phi_{t_{0}}(T)\right) .
$$

The right side can be estimated by

$$
\begin{aligned}
\left\|y^{\prime}\right\|_{L^{\infty}\left(t_{0}, T ; L^{2}(\Omega)\right)}\left\{\left\|\phi_{t_{0}}^{\prime}\right\|_{L^{1}\left(0, T ; L^{2}(\Omega)\right)}+\|\phi\|_{L^{\infty}\left(0, T ; L^{2}(\Omega)\right)}\right\} & \\
\leq\left\|y^{\prime}\right\|_{L^{\infty}\left(t_{0}, T ; L^{2}(\Omega)\right)}\left\{\left\|\phi^{\prime}\right\|_{L^{1}\left(0, T ; L^{2}(\Omega)\right)}+\right. & \left.\|\phi\|_{L^{\infty}\left(0, T ; L^{2}(\Omega)\right)}\right\} \\
& \leq C\left|s^{0}\right|\left\|y^{\prime}\right\|_{L^{\infty}\left(t_{0}, T ; L^{2}(\Omega)\right)}, \text { by Theorem } 2.1 .
\end{aligned}
$$

It follows then that

$$
\left|\sigma_{1}^{\prime}\left(t_{0}\right)+\int_{\Gamma} \psi_{1}\left(t_{0}\right) n\right| \leq C\left\|y^{\prime}\right\|_{L^{\infty}\left(t_{0}, T ; L^{2}(\Omega)\right)} .
$$

Since $t_{0}$ is arbitrary, the proof of the estimate is complete.

Step 4. Since $y \in C^{1}\left([0, T] ; L^{2}(\Omega)\right) \cap H^{2}\left(0, T ; V^{\prime}\right)$, there exists a sequence $\left(y_{k}\right)_{k} \subset C^{1}\left([0, T] ; L^{2}(\Omega)\right) \cap$ $H^{2}\left(0, T ; L^{2}(\Omega)\right)$ converging to $y$ in $C^{1}\left([0, T] ; L^{2}(\Omega)\right)$. Let $\left(\psi_{k}, \sigma_{k}\right)$ be the solution to $(31)$ with the source term $f=-y_{k}^{\prime \prime}$. Due to the estimates proved in steps 2 and $3,\left(\psi_{k}, \psi_{k}^{\prime}, \sigma_{k}, \sigma_{k}^{\prime}+\int_{\Gamma} \psi_{k} n\right)_{k}$ and $\left(\psi_{k}, \psi_{k}^{\prime}, \sigma_{k}, \sigma_{k}^{\prime}\right)_{k}$ are Cauchy sequences in $C([0, T] ; F)$, respectively converging to $\left(\psi_{1}, \psi_{1}^{\prime}, \sigma_{1}, \sigma_{1}^{\prime}+\int_{\Gamma} \psi_{1} n\right)$ and to $\left(\psi_{1}, \psi_{1}^{\prime}, \sigma_{1}, \sigma_{1}^{\prime}\right)$ in $C([0, T] ; F)$. Thus $\left(\psi_{1}, \psi_{1}^{\prime}, \sigma_{1}, \sigma_{1}^{\prime}+\int_{\Gamma} \psi_{1} n\right)$ and $\left(\psi_{1}, \psi_{1}^{\prime}, \sigma_{1}, \sigma_{1}^{\prime}\right)$ belong to $C([0, T] ; F)$.

Step 5. We have already seen in Section 3 that the Green formula is valid between the finite energy solution $(\phi, s)$ and the weak solution $(\psi, \sigma)$ : see the remark at the end of Section 3.

Remark 6.4. Since $\left(\psi_{1}, \psi_{1}^{\prime}, \sigma_{1}, \sigma_{1}^{\prime}\right)$ belongs to $C([0, T] ; F)$, then $\psi_{1}^{\prime} \in C\left([0, T] ; V^{\prime}\right)$.

Remark 6.5. From the estimates provided by the above theorem, it follows that the individual terms

$$
\sigma_{1}^{\prime} \in L^{\infty}\left(0, T ; \mathbb{R}^{2}\right) \quad \text { and } \quad \int_{\Gamma} \psi_{1} n \in L^{\infty}\left(0, T ; \mathbb{R}^{2}\right)
$$

are in the space $L^{\infty}\left(0, T ; \mathbb{R}^{2}\right)$. This is an additional regularity property for weak solutions $\left(\psi_{1}, \sigma_{1}\right)$ of $(31)$ which has a non-zero source term but has zero initial data. If the initial data is non-zero, as remarked in Section 4 , the above individual terms are not well-defined in general but only their sum is.

To define solutions to equation (31) by the transposition method with the source term $f=y^{\prime}(T) \otimes \delta_{T}-$ $y^{\prime}(0) \otimes \delta_{0}$, we cannot use test functions $(\phi, s)$ for which $\left(\phi(0), \phi^{\prime}(0), s(0), s^{\prime}(0)\right)=0$ because in that case the weak solution is only defined in the time interval $(0, T]$ and the source term $y^{\prime}(0) \otimes \delta_{0}$ is not taken into account in this interval. To take into account this source term in a weak formulation satisfied by a pair $(\psi, \sigma)$ we have to give a meaning to $\psi^{\prime}(0)$.

Definition 6.6. A function $(\psi, \sigma) \in L^{\infty}\left(0, T ; L^{2}(\Omega)\right) \times L^{\infty}\left(0, T ; \mathbb{R}^{2}\right)$ is a weak solution to equation (31) with the source term $f=y^{\prime}(T) \otimes \delta_{T}-y^{\prime}(0) \otimes \delta_{0}$ if $\psi^{\prime}$, the vector-valued distributional derivative of $\psi$ in $(0, T)$, 
belongs to $L^{\infty}\left(0, T ; V^{\prime}\right)$ and may be identified with a function in $B V\left([0, T] ; V^{\prime}\right)$ and if

$$
\int_{Q} \psi u+\int_{0}^{T} \sigma \cdot v=\left(y^{\prime}(T), \phi(T)\right)_{L^{2}(\Omega)}-\left(y^{\prime}(0), \phi^{0}\right)_{L^{2}(\Omega)}+\left\langle\phi^{0}, \psi^{\prime}(0)\right\rangle_{V, V^{\prime}}
$$

for all $u \in L^{1}\left(0, T ; L^{2}(\Omega)\right), v \in L^{1}\left(0, T ; \mathbb{R}^{2}\right), \phi^{0} \in V,\left(\phi^{1}, s^{0}, s^{1}\right)=0$, where $(\phi, s)$ is the finite energy solution to equation (3).

For the definition of the space $B V\left([0, T] ; V^{\prime}\right)$ we refer to [2] or to [12].

Theorem 6.7. Consider the system (31) with the source term $f=y^{\prime}(T) \otimes \delta_{T}-y^{\prime}(0) \otimes \delta_{0}$. Then there exists a unique weak solution $\left(\psi_{2}, \sigma_{2}\right)$ satisfying

$$
\left\|\psi_{2}\right\|_{L^{\infty}(0, T ; V)}+\left\|\psi_{2}^{\prime}\right\|_{L^{\infty}\left(0, T ; L^{2}(\Omega)\right)}+\left\|\sigma_{2}\right\|_{L^{\infty}\left(0, T ; \mathbb{R}^{2}\right)}+\left\|\sigma_{2}^{\prime}\right\|_{L^{\infty}\left(0, T ; \mathbb{R}^{2}\right)} \leq C\left\|y^{\prime}\right\|_{L^{\infty}\left(0, T ; L^{2}(\Omega)\right)} .
$$

Moreover $\psi_{2} \in C\left([0, T] ; V_{w}\right), \sigma_{2} \in C^{1}\left([0, T] ; \mathbb{R}^{2}\right)$ and the following formula holds

$$
\begin{aligned}
\int_{Q} \psi_{2} u+\int_{0}^{T} \sigma_{2} \cdot v=\left(y^{\prime}(T), \phi(T)\right)_{L^{2}(\Omega)}-\left(y^{\prime}(0), \phi(0)\right)_{L^{2}(\Omega)}+\left\langle\phi^{0}, \psi_{2}^{\prime}(0)\right\rangle_{V_{,} V^{\prime}} & \\
& -\left(\phi^{1}, \psi_{2}(0)\right)_{L^{2}(\Omega)}+s^{0} \cdot\left(\sigma_{2}^{\prime}(0)+\int_{\Gamma} \psi_{2}(0) n\right)-\left(s^{1}+\int_{\Gamma} \phi^{0} n\right) \cdot \sigma_{2}(0),
\end{aligned}
$$

for all $u \in L^{1}\left(0, T ; L^{2}(\Omega)\right), v \in L^{1}\left(0, T ; \mathbb{R}^{2}\right)$, and $\left(\phi^{0}, \phi^{1}, s^{0}, s^{1}\right) \in Z$, where $(\phi, s)$ is the finite energy solution to equation (3). The space $V_{w}$ denotes the space $V$ endowed with its weak topology, and $C\left([0, T] ; V_{w}\right)$ is the space of continuous functions from $[0, T]$ into $V_{w}$.

Proof. The uniqueness is obvious. To prove the existence we proceed by approximation. Let $\left(\mu_{k}\right)_{k}$ be a sequence in $L^{1}\left(0, T ; L^{2}(\Omega)\right)$ converging to $y^{\prime}(T) \otimes \delta_{T}-y^{\prime}(0) \otimes \delta_{0}$ for the weak-star topology of $\mathcal{M}\left([0, T] ; L^{2}(\Omega)\right)$, and satisfying

$$
\left\|\mu_{k}\right\|_{L^{1}\left(0, T ; L^{2}(\Omega)\right)} \leq\left\|y^{\prime}(T)\right\|_{L^{2}(\Omega)}+\left\|y^{\prime}(0)\right\|_{L^{2}(\Omega)} .
$$

Let $\left(\psi_{k}, \sigma_{k}\right)$ be the finite energy solution of the equation

$$
\begin{array}{lll}
\psi^{\prime \prime}-\Delta \psi=\mu_{k} & & \text { in } Q, \\
\psi=0 & \text { on } \Sigma_{e}, \\
\partial_{n} \psi=\sigma^{\prime} \cdot n & \text { on } \Sigma, \\
\psi(T)=0 \quad \text { and } & \psi^{\prime}(T)=0 & \text { in } \Omega, \\
\sigma^{\prime \prime}+\sigma=-\int_{\Gamma} \psi^{\prime} n & & \text { in }(0, T), \\
\sigma(T)=0 \quad \text { and } & \sigma^{\prime}(T)=0 & \text { in } \mathbb{R}^{2} .
\end{array}
$$

From Theorem 3.1, we have the estimate

$$
\left\|\left(\psi_{k}, \psi_{k}^{\prime}, \sigma_{k}, \sigma_{k}^{\prime}\right)\right\|_{C([0, T] ; Z)} \leq C\left\|\mu_{k}\right\|_{L^{1}\left(0, T ; L^{2}(\Omega)\right)} \leq C\left\|y^{\prime}\right\|_{L^{\infty}\left(0, T ; L^{2}(\Omega)\right)} .
$$

Furthermore, integration by parts between $(\phi, s)$ and $\left(\psi_{k}, \sigma_{k}\right)$ yields

$$
\begin{aligned}
\int_{Q} u \psi_{k}+\int_{0}^{T} v \cdot \sigma_{k}=\int_{Q} \mu_{k} \phi-\left(\phi^{1}, \psi_{k}(0)_{L^{2}(\Omega)}+\left\langle\phi^{0}, \psi_{k}^{1}(0)\right\rangle_{V, V^{\prime}}\right. \\
\\
\quad+s^{0} \cdot\left(\sigma_{k}^{\prime}(0)+\int_{\Gamma} \psi_{k}(0) n\right)-\left(s^{1}+\int_{\Gamma} \phi^{0} n\right) \cdot \sigma_{k}(0) .
\end{aligned}
$$


For a subsequence, we have

$$
\begin{gathered}
\left(\psi_{k}, \psi_{k}^{\prime}, \sigma_{k}, \sigma_{k}^{\prime}\right) \rightarrow\left(\psi_{2}, \psi_{2}^{\prime}, \sigma_{2}, \sigma_{2}^{\prime}\right) \text { in } L^{\infty}(0, T ; Z) \text { weak } *, \\
\left(\psi_{k}(0), \psi_{k}^{\prime}(0), \sigma_{k}(0), \sigma_{k}^{\prime}(0)\right) \rightarrow\left(\psi_{2}^{0}, \psi_{2}^{1}, \sigma_{2}^{0}, \sigma_{2}^{1}\right) \text { in } Z \text { weak. }
\end{gathered}
$$

Furthermore, the boundary quantities admit stronger estimates:

$$
\begin{aligned}
\left\|\left(\psi_{2}^{0}, \psi_{2}^{1}, \sigma_{2}^{0}, \sigma_{2}^{1}\right)\right\|_{Z} & \leq \lim \inf _{k \rightarrow \infty}\left\|\left(\psi_{k}(0), \psi_{k}^{\prime}(0), \sigma_{k}(0), \sigma_{k}(0), \sigma_{k}^{\prime}(0)\right)\right\|_{Z} \\
& \leq C\left\|y^{\prime}\right\|_{L^{\infty}\left(0, T ; L^{2}(\Omega)\right)} .
\end{aligned}
$$

Since $\psi \in L^{\infty}(0, T ; V)$ and $\psi^{\prime} \in L^{\infty}\left(0, T ; L^{2}(\Omega)\right)$, it follows first that $\psi \in C\left([0, T] ; L^{2}(\Omega)\right)$, and next that $\psi \in$ $C\left([0, T] ; V_{w}\right)$ (we can apply [7], Chap. 18, Lem. 5.6). A similar argument applied to $\sigma$ yields $\sigma \in C\left([0, T] ; \mathbb{R}^{2}\right)$. Next using the equation satisfied by $\sigma$ and the facts that $\psi \in C\left([0, T] ; V_{w}\right)$ and $\sigma \in C\left([0, T] ; \mathbb{R}^{2}\right)$, we obtain $\sigma^{\prime} \in C\left([0, T] ; \mathbb{R}^{2}\right)$. Observe that $\int_{\Gamma} \psi(\cdot) n \in C\left([0, T] ; \mathbb{R}^{2}\right)$. Thus we have $\left(\psi_{2}^{0}, \sigma_{2}^{0}, \sigma_{2}^{1}, \sigma_{2}^{1}+\int_{\Gamma} \psi_{2}^{0} n\right)=$ $\left(\psi_{2}(0), \sigma_{2}(0), \sigma_{2}^{\prime}(0), \sigma_{2}^{\prime}(0)+\int_{\Gamma} \psi_{2}(0) n\right)$. Since

$$
\left\|\left(\psi_{k}, \psi_{k}^{\prime}, \sigma_{k}, \sigma_{k}^{\prime}\right)\right\|_{C([0, T] ; Z)} \leq C\left\|y^{\prime}\right\|_{L^{\infty}\left(0, T ; L^{2}(\Omega)\right)},
$$

using the equation satisfied by $\psi_{k}$, we prove that

$$
\left\|\psi_{k}^{\prime}\right\|_{B V\left([0, T] ; V^{\prime}\right)} \leq C\left\|y^{\prime}\right\|_{L^{\infty}\left(0, T ; L^{2}(\Omega)\right)} .
$$

Thus $\psi_{2}^{\prime} \in B V\left([0, T] ; V^{\prime}\right)$, and

$$
\psi_{k}^{\prime}(0) \rightarrow \psi_{2}^{\prime}(0) \text { weakly in } V^{\prime},
$$

(see [2], Th. 3.5 or [12], Prop 16.1). Therefore $\psi_{2}^{\prime}(0)=\psi_{2}^{1}$.

We obtain (34) by passing to the limit in the Green formula satisfied by $\left(\psi_{k}, \sigma_{k}\right)$.

Combining Definitions 6.2 and 6.6, we state the following

Definition 6.8. A function $(\psi, \sigma) \in L^{\infty}\left(0, T ; L^{2}(\Omega)\right) \times L^{\infty}\left(0, T ; \mathbb{R}^{2}\right)$ is a weak solution to equation (31) with the source term $f=-d_{t}\left[y^{\prime}\right]$ if $\psi^{\prime}$, the vector-valued distributional derivative of $\psi$ in $(0, T)$, belongs to $L^{\infty}\left(0, T ; V^{\prime}\right)$ and may be identified with a function in $C\left([0, T] ; V^{\prime}\right)+B V\left([0, T] ; V^{\prime}\right)$ and if

$$
\int_{Q} \psi u+\int_{0}^{T} \sigma \cdot v=\int_{Q} y^{\prime} \phi^{\prime}+\left\langle\phi^{0}, \psi^{\prime}(0)\right\rangle_{V, V^{\prime}}
$$

for all $u \in L^{1}\left(0, T ; L^{2}(\Omega)\right), v \in L^{1}\left(0, T ; \mathbb{R}^{2}\right), \phi^{0} \in V,\left(\phi^{1}, s^{0}, s^{1}\right)=0$, where $(\phi, s)$ is the finite energy solution to equation $(3)$.

Theorem 6.9. The equation (31) with $f=-d_{t}\left[y^{\prime}\right]$ admits a unique weak solution $(\psi, \sigma)$ in $L^{\infty}\left(0, T ; L^{2}(\Omega)\right) \times$ $L^{\infty}\left(0, T ; \mathbb{R}^{2}\right)$ in the sense of definition 6.8 and it satisfies the estimate

$$
\|\psi\|_{L^{\infty}\left(0, T ; L^{2}(\Omega)\right)}+\left\|\psi^{\prime}\right\|_{L^{\infty}\left(0, T ; V^{\prime}\right)}+\|\sigma\|_{L^{\infty}\left(0, T ; \mathbb{R}^{2}\right)}+\left\|\sigma^{\prime}\right\|_{L^{\infty}\left(0, T ; \mathbb{R}^{2}\right)} \leq C\left\|y^{\prime}\right\|_{L^{\infty}\left(0, T ; L^{2}(\Omega)\right)} .
$$

There exists a unique $\left(\psi^{0}, \psi^{1}, \sigma^{0}, \tilde{\sigma}^{1}\right)$ in $L^{2}(\Omega) \times V^{\prime} \times \mathbb{R}^{2} \times \mathbb{R}^{2}$ such that

$$
\int_{Q} \psi u+\int_{0}^{T} \sigma \cdot v=\int_{Q} y^{\prime} \phi^{\prime}-\left(\phi^{1}, \psi^{0}\right)_{L^{2}(\Omega)}+\left\langle\phi^{0}, \psi^{1}\right\rangle_{V, V^{\prime}}+s^{0} \cdot \tilde{\sigma}^{1}-\left(s^{1}+\int_{\Gamma} \phi^{0} n\right) \cdot \sigma^{0},
$$

for all $u \in L^{1}\left(0, T ; L^{2}(\Omega)\right), v \in L^{1}\left(0, T ; \mathbb{R}^{2}\right)$, and $\left(\phi^{0}, \phi^{1}, s^{0}, s^{1}\right) \in Z$, where $(\phi, s)$ is the finite energy solution to equation (3). Moreover the following estimate holds

$$
\left\|\psi^{0}\right\|_{L^{2}(\Omega)}+\left\|\psi^{1}\right\|_{V^{\prime}}+\left|\sigma^{0}\right|+\left|\tilde{\sigma}^{1}\right| \leq C\left\|y^{\prime}\right\|_{L^{\infty}\left(0, T ; L^{2}(\Omega)\right)} .
$$


Proof. The uniqueness of a weak solution is obvious. The existence of a solution and its estimate follow from Theorems 6.3 and 6.7 .

The uniqueness of $\left(\psi^{0}, \psi^{1}, \sigma^{0}, \tilde{\sigma}^{1}\right) \in L^{2}(\Omega) \times V^{\prime} \times \mathbb{R}^{2} \times \mathbb{R}^{2}$ obeying (36) follows from the fact that the mapping $\left(\phi^{0}, \phi^{1}, s^{0}, s^{1}\right) \mapsto\left(\phi^{0}, \phi^{1}, s^{0}, s^{1}+\int_{\Gamma} \phi^{0} n\right)$ is an isomorphism from $Z$ into itself.

Following the decomposition $(\psi, \sigma)=\left(\psi_{1}, \sigma_{1}\right)+\left(\psi_{2}, \sigma_{2}\right)$, the problem of existence of $\left(\psi^{0}, \psi^{1}, \sigma^{0}, \widetilde{\sigma}^{\prime}\right)$ can be reduced to two problems: one involving $\left(\psi_{1}, \sigma_{1}\right)$ and another one for $\left(\psi_{2}, \sigma_{2}\right)$. These two problems are already studied in the previous two theorems.

Remark 6.10. We cannot prove that

$$
\left\|\psi^{\prime}\right\|_{L^{\infty}\left(0, T ; V^{\prime}\right)} \leq C\left\|y^{\prime}\right\|_{L^{2}\left(0, T ; L^{2}(\Omega)\right)},
$$

because, in Theorem 2.5, we were not able to establish the estimate

$$
\left\|\phi^{\prime}\right\|_{L^{2}\left(0, T ; L^{2}(\Omega)\right)} \leq C\left\|f_{1}\right\|_{L^{1}(0, T ; V)} .
$$

So far, we have been dealing with weak solutions of (31) where we have zero end conditions at time $T$. Quite obviously, we can do the same thing with weak solutions of (1) with zero initial conditions at time $t=0$. Thus in the case when $u=-d_{t}\left[y^{\prime}\right]$, and when $\left(\phi^{0}, \phi^{1}, s^{0}, s^{1}\right)=0$ we can define the solution to equation (1) by transposition, in a similar way (with obvious modifications) as in Definition 6.2. Therefore, combining the results of Section 3 and of the present section, we can assert that if $u=-d_{t}\left[y^{\prime}\right]$ and if $\left(\phi^{0}, \phi^{1}, s^{0}, s^{1}\right) \in Y$, equation (1) admits a unique weak solution $(\phi, s) \in L^{\infty}\left(0, T ; L^{2}(\Omega)\right) \times L^{\infty}\left(0, T ; \mathbb{R}^{2}\right)$.

Theorem 6.11. Let $(\psi, \sigma) \in L^{\infty}\left(0, T ; L^{2}(\Omega)\right) \times L^{\infty}\left(0, T ; \mathbb{R}^{2}\right)$ be the unique solution of equation (31), and let $\left(\psi^{0}, \psi^{1}, \sigma^{0}, \tilde{\sigma}^{1}\right) \in F$ be defined by the formula (36). Then $(\psi, \sigma) \in L^{\infty}\left(0, T ; L^{2}(\Omega)\right) \times L^{\infty}\left(0, T ; \mathbb{R}^{2}\right)$ is the unique weak solution of equation (1) corresponding to $u=-d_{t}\left[y^{\prime}\right]$ and to $\left(\phi^{0}, \phi^{1}, s^{0}, s^{1}\right)=(\widetilde{J})^{-1}\left(\psi^{0}, \psi^{1}, \sigma^{0}, \tilde{\sigma}^{1}\right)$.

Proof. We use the notation in the proof of Theorem 6.7. We can easily verify that the solution $\left(\psi_{k}, \sigma_{k}\right)$ of equation (35) is also the solution $\left(\phi_{k}, s_{k}\right)$ of equation (1) corresponding to $u=\mu_{k}$ and to $\left(\phi^{0}, \phi^{1}, s^{0}, s^{1}\right)=$ $\left(\psi_{k}(0), \psi_{k}^{\prime}(0), \sigma_{k}(0), \sigma_{k}^{\prime}(0)\right)$. By definition of $\widetilde{J}$, we have $\left(\psi_{k}(0), \psi_{k}^{\prime}(0), \sigma_{k}(0), \sigma_{k}^{\prime}(0)\right)=$ $(\widetilde{J})^{-1}\left(\psi_{k}(0), \psi_{k}^{\prime}(0), \sigma_{k}(0), \sigma_{k}^{\prime}(0)+\int_{\Gamma} \psi_{k}(0) n\right)$. Therefore the theorem is obtained by passing to the limit when $k \rightarrow \infty$.

\section{Proof of Theorem 6.1}

Step 1. We define the operator $\widehat{\Lambda}$ from $Z$ into $Z^{\prime}$ by

$$
\widehat{\Lambda}\left(y^{0}, y^{1}, r^{0}, r^{1}\right)=\left(-\psi^{1}, \psi^{0},-\tilde{\sigma}^{1}, \sigma^{0}\right),
$$

where $(\psi, \sigma)$ is the weak solution to equation $(31),\left(\psi^{0}, \psi^{1}, \sigma^{0}, \tilde{\sigma}^{1}\right) \in L^{2}(\Omega) \times V^{\prime} \times \mathbb{R}^{2} \times \mathbb{R}^{2}$ is associated with $(\psi, \sigma)$ by the formula $(36)$, and $(y, r)$ is the finite energy solution of equation (21). We define the isomorphism $\widehat{J}$ from $Z$ onto $Z$ by

$$
\widehat{J}\left(\phi^{0}, \phi^{1}, s^{0}, s^{1}\right)=\left(\phi^{0}, \phi^{1}, s^{0}, s^{1}+\int_{\Gamma} \phi^{0} n\right) .
$$

From the definition of $\widehat{\Lambda}$ and formula (36) it follows that

$$
\left\langle\widehat{\Lambda}\left(y^{0}, y^{1}, r^{0}, r^{1}\right), \widehat{J}\left(\phi^{0}, \phi^{1}, s^{0}, s^{1}\right)\right\rangle_{Z^{\prime}, Z}=\int_{Q} y^{\prime} \phi^{\prime} .
$$

Due to Theorems 2.1 and 4.5, the mapping

$$
\left(\left(y^{0}, y^{1}, r^{0}, r^{1}\right),\left(\phi^{0}, \phi^{1}, s^{0}, s^{1}\right)\right) \longmapsto \int_{Q} y^{\prime} \phi^{\prime}
$$


defines an inner product on $Z$ whose norm is equivalent to the original norm in $Z$. This observation and the above identity implies that $\widehat{J}^{*} \widehat{\Lambda}$ is an isomorphism from $Z$ onto $Z^{\prime}$. Since $\widehat{J}^{*}$ is an isomorphism from $Z^{\prime}$ onto $Z^{\prime}$, we deduce that $\widehat{\Lambda}$ is an isomorphism from $Z$ onto $Z^{\prime}$.

Step 2. Let us now start with $\left(\phi^{0}, \phi^{1}, s^{0}, s^{1}\right)$ in $Y$. Let us set $\left(\phi^{0}, \phi^{1}, s^{0}, \tilde{s}^{1}\right)=\widetilde{J}\left(\phi^{0}, \phi^{1}, s^{0}, s^{1}\right)$, where $\widetilde{J}$ is the operator defined in Theorem 5.1 and observe that $\left(-\phi^{1}, \phi^{0},-\tilde{s}^{1}, s^{0}\right)$ belongs to $Z^{\prime}$. Let $\left(y^{0}, y^{1}, r^{0}, r^{1}\right) \in Z$ be the solution to the equation

$$
\widehat{\Lambda}\left(y^{0}, y^{1}, r^{0}, r^{1}\right)=\left(-\phi^{1}, \phi^{0},-\tilde{s}^{1}, s^{0}\right) .
$$

If $(y, r)$ is the finite energy solution to equation (21) corresponding to $\left(y^{0}, y^{1}, r^{0}, r^{1}\right)$ and $(\psi, \sigma)$ is the weak solution to (31), then from the definition of $\widehat{\Lambda}$ it follows that

$$
\left(\psi^{0}, \psi^{1}, \sigma^{0}, \tilde{\sigma}^{1}\right)=\widetilde{J}\left(\phi^{0}, \phi^{1}, s^{0}, s^{1}\right) .
$$

Therefore if we set $u=-d_{t}\left[y^{\prime}\right]$, and $\left(\phi^{0}, \phi^{1}, s^{0}, s^{1}\right)=(\widetilde{J})^{-1}\left(\psi^{0}, \psi^{1}, \sigma^{0}, \tilde{\sigma}^{1}\right)$, due to Theorem 6.11 the solution $(\phi, s)$ to equation (1) obeys $\phi(T)=\phi^{\prime}(T)=0$ and $s(T)=s^{\prime}(T)=0$, and Theorem 6.1 is proved.

Acknowledgements. The authors thank an anonymous referee for helpful questions and comments on a preliminary version of this paper.

\section{REFERENCES}

[1] G. Avalos, I. Lasiecka, Exact controllability of structural acoustic interactions. J. Math. Pures Appl. 82 (2003) $1047-1073$.

[2] V. Barbu, T. Precupanu, Convexity and Optimization in Banach Spaces, 2nd ed., D. Reidel, Dordrecht (1986).

[3] A. Bensoussan, G. Da Prato, M.C. Delfour and S.K. Mitter, Representation and Control of Infinite Dimensional Systems. Birkhäuser, Boston 1 (1992).

[4] C. Conca, J. Planchard, B. Thomas and M. Vanninathan, Problèmes mathématiques en couplage fluide-structure. Eyrolles, Paris (1994).

[5] C. Conca, J. Planchard and M. Vanninathan, Fluids and periodic structures. Masson and J. Wiley, Paris (1995).

[6] L. Cot, J.-P. Raymond and J. Vancostenoble, Exact controllability of an aeroacoustic model. In preparation.

[7] R. Dautray and J.-L. Lions, Analyse Mathématique et Calcul Scientifique. Masson, Paris (1987).

[8] P. Destuynder and E. Gout d'Henin, Existence and uniqueness of a solution to an aeroacoustic model. Chin. Ann. Math. 23B (2002) 11-24.

[9] E. Gout d'Henin, Ondes de Stoneley en interaction fluide-structure. Ph.D. Thesis, Université de Poitiers (2002).

[10] J.-L. Lions, Contrôlabilité exacte, perturbations et stabilisation de systèmes distribués. Masson, Paris (1988).

[11] S. Micu and E. Zuazua, Boundary controllability of a linear hybrid system arising in the control of noise. SIAM J. Control Optim. 35 (1997) 531-555.

[12] J.J. Moreau, Bounded variation in time, in Topics in Nonsmooth Mechanics, J.J. Moreau, P.D. Panagiotopoulos, G. Strang Eds. Birkhäuser, Boston (1988) 1-74. 